\title{
METASTABILITY AND PINNING FOR CONVECTION-DIFFUSION-REACTION EQUATIONS IN THIN DOMAINS*
}

\author{
XIAODI SUN ${ }^{\dagger}$ AND MICHAEL J. WARD ${ }^{\dagger}$
}

\begin{abstract}
Two singularly perturbed convection-diffusion-reaction equations are examined to show the effect of small spatial inhomogeneities on metastable dynamics in one spatial dimension. The two problems that are considered are the Ginzburg-Landau equation from phase separation theory and a viscous shock problem modeling transonic nozzle flow. For each problem, the differential operator is perturbed by an exponentially small spatially inhomogeneous term as the singular perturbation parameter $\varepsilon$ tends to zero. This weak spatially inhomogeneous term represents the perturbing effect on the metastable dynamics of an internal layer that is slowly propagating along a channel of slowly varying cross-sectional area. It is shown that the effect of such a perturbation can be very significant and often leads to the existence of new stable equilibrium internal layer solutions that do not exist in the absence of the perturbation. This pinning effect induced by the perturbation is studied asymptotically as $\varepsilon \rightarrow 0$ and the asymptotic results are compared with full numerical results.
\end{abstract}

1. Introduction. We study two singularly perturbed evolution equations exhibiting metastable dynamics in a weakly inhomogeneous medium. The first problem we consider is the following generalized Ginzburg-Landau equation, which models the slow propagation of an internal layer in a thin channel:

$$
\begin{aligned}
u_{t} & =\frac{\varepsilon^{2}}{A}\left(A u_{x}\right)_{x}+Q(u), \quad 0<x<1, \quad t>0, \\
u_{x}(0, t) & =u_{x}(1, t)=0, \quad u(x, 0)=u_{0}(x) .
\end{aligned}
$$

Here $\varepsilon>0$ is a small parameter and $A=A(x, \varepsilon)>0$ is the local cross-sectional area of the channel, which is specified below. In addition, $Q(u)$ is a smooth function with exactly three zeroes on the interval $\left[s_{-}, s_{+}\right]$located at $u=s_{-}<0, u=0$, and $u=s_{+}>0$. Introducing the double-well potential $V(u)$ by $V(u) \equiv-\int_{s_{-}}^{u} Q(\eta) d \eta$, we assume that

$$
Q^{\prime}\left(s_{ \pm}\right)<0, \quad Q^{\prime}(0)>0, \quad V\left(s_{+}\right)=0 .
$$

A typical example is $Q(u)=2\left(u-u^{3}\right)$ for which $s_{ \pm}= \pm 1$ and $V(u)=\frac{1}{2}\left(1-u^{2}\right)^{2}$.

The motivation for studying (1.1) is related to the problem of determining the conditions for the existence of stable spatially inhomogeneous steady-state solutions to the Ginzburg-Landau equation

$$
u_{t}=\triangle u+Q(u), \quad x \in D ; \quad \partial_{n} u=0, \quad x \in \partial D .
$$

Here $D$ is a bounded domain in $R^{N}$ and $\partial_{n}$ denotes the outward normal derivative to $\partial D$. In a convex domain, it is well known that (1.3) does not admit a stable spatially inhomogeneous steady-state solution (cf. [5], [18]). However, this non-existence result does not hold for non-convex domains (cf. [9], [12], [18]). In Appendix A, we show how (1.1) arises from an asymptotic reduction of (1.3) when $D$ is a thin, axially symmetric domain as shown in Figure 1.1. In this context, $x$ represents the direction along the axis of the channel and $A$ represents the local cross-sectional area of the channel.

*Received August 7, 1998; revised November 25, 1998.

${ }^{\dagger}$ Department of Mathematics, University of British Columbia, Vancouver, B.C. V6T 1Z2, Canada (ward@math.ubc.ca). This work was supported by NSERC grant 5-81541. 
When $A \equiv 1$, which yields a slab geometry, it is well known that the propagation of an internal layer for (1.1) is exponentially slow as $\varepsilon \rightarrow 0$ (i.e., metastable) and that a stable spatially inhomogeneous solution for (1.1) does not exist (see [4], [8], [14], [22]). When $A \equiv 1$, the metastability is a consequence of an exponentially small eigenvalue for the linearization of (1.1) around an internal layer solution.

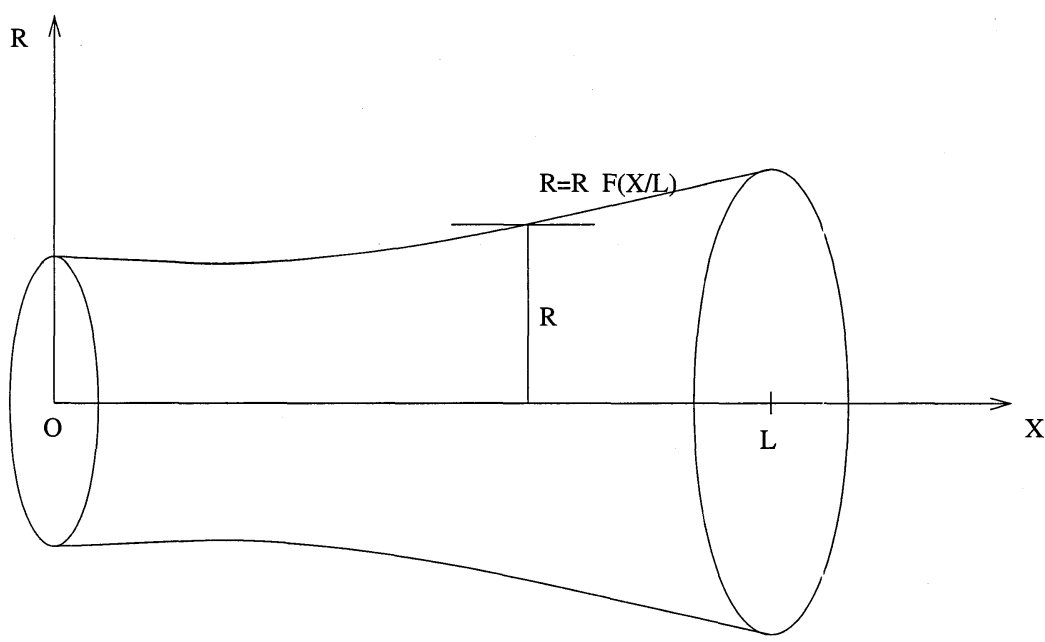

FIG. 1.1. A cylinder of revolution with cross-section described in dimensional variables by $R=R_{0} F(X / L)$.

This exponential ill-conditioning suggests that the dynamics of an internal layer solution for (1.1) will depend very sensitively on the channel cross-section $A$ when $A$ is slightly offset from the uniform value $A \equiv 1$. In particular, exponentially small changes in $A-1$ should influence the dynamics greatly. Therefore, in $\S 2$, we study (1.1) as $\varepsilon \rightarrow 0$ for an $A(x ; \varepsilon)$ of the form

$$
A(x ; \varepsilon)=1+\varepsilon^{\mu} g(x) e^{-d / \varepsilon} .
$$

Here $\mu$ and $d>0$ are constants and $g(x)$ is smooth. If $g^{\prime \prime}(x)<0$, then $D$ is convex and we expect that (1.1) will have no stable spatially inhomogeneous equilibrium solutions. When $g^{\prime \prime}(x)>0$ and $0<d<d_{c}$, where $d_{c}$ is some constant, we show in $\S 2$ that $(1.1)$ can have a stable spatially inhomogeneous equilibrium internal layer solution where the internal layer is located at a zero of $g^{\prime}(x)$. This phenomenon, in which an internal layer or other localized structure is stabilized by a weakly inhomogeneous medium, is called pinning. The effect of pinning of other localized structures such as vortices in superconductivity has been studied in [6], [16]. When $g^{\prime \prime}(x)>0$ and $d=d_{c}$, we show in $\S 2$ that the internal layer can be pinned at other locations in the interval $[0,1]$. In $\S 2.1$, we provide an asymptotic estimate for the principal eigenvalue $\lambda_{0}$ associated with the linearization of (2.1). In $\S 2.2$, we use the projection method, as surveyed in [23], to derive a differential equation for the location $x_{0}(t)$ of the internal layer, and we determine its limiting behavior as $t \rightarrow \infty$. Finally, in $\S 2.3$, we compare our asymptotic results with corresponding results obtained from a full numerical solution of (1.1). 
The second problem we consider is the nonlinear convection-diffusion equation

$$
\begin{array}{rlrl}
u_{t}+[f(u)]_{x}-c(x ; \varepsilon) h(u) & =\varepsilon u_{x x}, & 0<x<1, \quad t>0, \\
u(0)=\alpha_{-}, \quad u(1) & =\alpha_{+} ; \quad & u(x, 0) & =u_{0}(x) .
\end{array}
$$

Here $0<\varepsilon \ll 1, \alpha_{-}>0$ and $\alpha_{+}<0$ are constants, and $h(u)$ and $f(u)$ are smooth. The flux function $f(u)$ is assumed to be convex and satisfies

$$
f\left(\alpha_{+}\right)=f\left(\alpha_{-}\right), \quad f(0)=f^{\prime}(0)=0, \quad u f^{\prime}(u)>0 \text { for } u \neq 0 .
$$

The function $c(x ; \varepsilon)$ is chosen to be

$$
c(x ; \varepsilon)=-\varepsilon^{\mu} g^{\prime}(x) e^{-\varepsilon^{-1} d} .
$$

Here $\mu$ and $d>0$ are constants and $g(x)$ is smooth.

A primary motivation for studying (1.5)-(1.7) is that, for the special case when $h(u)=u$ and $f(u)=u^{2} / 2$, this problem models transonic gas flow in a nozzle of cross-sectional area $A(x ; \varepsilon)$ given by $c(x ; \varepsilon)=-A_{x}(x ; \varepsilon) / A(x ; \varepsilon)$ (cf. [11], [10], [17], [20]). Hence, for $\varepsilon \ll 1$, the cross-sectional area $A(x ; \varepsilon)$ can be taken precisely as in (1.4). In this context, the nozzle is said to be divergent if $g^{\prime}(x)>0$ for all $x$, convergent if $g^{\prime}(x)<0$ for all $x$, and convergent-divergent if $g^{\prime}(x)$ has no definite sign. For Burgers equation $\left(f(u)=u^{2} / 2\right)$ in a straight channel where $g(x) \equiv 0$, it was shown in [13], [15], and [19] that there exists a unique and stable equilibrium shock layer solution centered at $x_{0}=1 / 2$. It was also shown that for the corresponding time-dependent problem, a viscous shock, which gets formed from the initial data, tends toward the steady-state solution only over an asymptotically exponentially long time interval as $\varepsilon \rightarrow 0$. This metastable behavior arises from the occurrence of an asymptotically exponentially small principal eigenvalue for the linearization of Burgers equation around the viscous shock solution. In view of this exponential ill-conditioning of Burgers equation, we expect that shock-layer solutions can be significantly altered by perturbing the differential operator by exponentially small terms. In [15], we remark that the effect of exponentially small but spatially homogeneous perturbations was considered.

Our primary goal in $\S 3$ is to study the pinning effect induced by the spatially inhomogeneous term $c(x ; \varepsilon)$ in $(1.5)$. In particular, we analyze the existence, stability, and dynamics of equilibrium and time-dependent shock-layer solutions to (1.5). In $\S 3.1$, we obtain an asymptotic estimate for the principal eigenvalue $\lambda_{0}$ associated with the linearization of (1.5) around a shock-layer profile. In $\S 3.2$, we use the projection method of [23] to derive a differential equation for the location $x_{0}(t)$ of the shock-layer trajectory. We then analyze the equilibrium solutions of this differential equation and determine their stability properties. In $\S 3.3$, we illustrate the results for certain forms of $g(x)$ when $h(u)=u$ and $f(u)=u^{2} / 2$, modeling transonic nozzle flow, and we compare our asymptotic results with corresponding numerical results. Our results show that, under certain assumptions, there can exist stable steady-state shock-layer solutions along a convergent nozzle or in the convergent part of a convergent-divergent nozzle. In contrast, it was shown, using a nonlinear stability analysis in [7], that when $c(x ; \varepsilon)$ is independent of $\varepsilon$ and when the diffusive term $\varepsilon u_{x x}$ in (1.5) is absent, the corresponding inviscid problem does not admit stable shock waves in these nozzles.

2. A generalized Ginzburg-Landau equation. We now study (1.1) in the limit $\varepsilon \rightarrow 0$ with $A(x ; \varepsilon)$ as given in (1.4). A one-layer metastable pattern for (1.1) 
can be approximated by

$$
u(x, t) \sim u_{c}\left[\varepsilon^{-1}\left(x-x_{0}(t)\right)\right]
$$

where $u_{c}(z)$ is the heteroclinic orbit that connects $s_{+}$and $s_{-}$, which satisfies

$$
u_{c}^{\prime \prime}(z)+Q\left(u_{c}\right)=0, \quad-\infty<z<\infty, \quad u_{c}(0)=0
$$

$$
u_{c}(z) \sim s_{-}+a_{-} e^{\nu_{-} z}, \quad \text { as } \quad z \rightarrow-\infty ; \quad u_{c}(z) \sim s_{+}-a_{+} e^{-\nu_{+} z}, \quad \text { as } \quad z \rightarrow \infty .
$$

Here the positive constants $\nu_{ \pm}$and $a_{ \pm}$are defined by

$$
\nu_{ \pm}=\left[-Q^{\prime}\left(s_{ \pm}\right)\right]^{1 / 2}, \quad \log a_{ \pm}=\log \left( \pm s_{ \pm}\right)+\int_{0}^{s_{ \pm}}\left[\frac{ \pm \nu_{ \pm}}{[2 V(s)]^{1 / 2}}+\frac{1}{s-s_{ \pm}}\right] d s
$$

We now look for a solution to (1.1) for $t \gg 1$ in the form

$$
u(x, t)=u_{c}\left[\varepsilon^{-1}\left(x-x_{0}(t)\right)\right]+w(x, t)
$$

where $w \ll u_{c}$ and $w_{t} \ll \partial_{t} u_{c}$. The trajectory $x_{0}=x_{0}(t)$ gives the approximate location of the zero of $u(x, t)$ during the metastable evolution. Substituting (2.4) into (1.1), and using (2.2), we obtain that $w$ satisfies the quasi-steady problem

$$
\begin{aligned}
L_{\varepsilon} w & =A\left(-\varepsilon^{-1} x_{0}^{\prime} u_{c}^{\prime}(z)-\varepsilon \frac{A_{x}}{A} u_{c}^{\prime}\right), \quad 0<x<1, \\
w_{x}(0, t) & =u_{x}(0, t)-\left.\partial_{x} u_{c}\right|_{x=0} \sim-\varepsilon^{-1} a_{-} \nu_{-} e^{-\nu^{\prime}-x_{0} / \varepsilon}, \\
w_{x}(1, t) & =u_{x}(1, t)-\left.\partial_{x} u_{c}\right|_{x=1} \sim-\varepsilon^{-1} a_{+} \nu_{+} e^{-\nu_{+}\left(1-x_{0}\right) / \varepsilon}
\end{aligned}
$$

where $z=\varepsilon^{-1}\left[x-x_{0}(t)\right]$. Here $A$ is given in (1.4) and the operator $L_{\varepsilon}$ is defined by

$$
L_{\varepsilon} w=\varepsilon^{2}\left(A w_{x}\right)_{x}+A Q^{\prime}\left(u_{c}\right) w .
$$

2.1. The eigenvalue analysis. For a fixed $x_{0} \in(0,1)$, we now study the eigenvalue problem

$$
\begin{aligned}
L_{\varepsilon} \phi & =\lambda \phi, \quad 0<x<1, \\
\phi_{x}(0) & =\phi_{x}(1)=0, \quad(\phi, \phi)=1 .
\end{aligned}
$$

Here $(u, v) \equiv \int_{0}^{1} u v d x$. For this eigenproblem, the eigenvalues $\lambda_{j}$ for $j \geq 0$ are real and the principal eigenvalue $\lambda_{0}$ is exponentially small as $\varepsilon \rightarrow 0$. To estimate $\lambda_{0}$ and the corresponding eigenfunction $\phi_{0}$, we use the trial function $\tilde{\phi}_{0} \equiv u_{c}^{\prime}\left[\varepsilon^{-1}\left(x-x_{0}\right)\right]$. Then, upon integrating by parts, we derive

$$
\lambda_{0}\left(\phi_{0}, \tilde{\phi}_{0}\right)=\left(\phi_{0}, L_{\varepsilon} \tilde{\phi}_{0}\right)-\left.\varepsilon^{2} A \phi_{0} \tilde{\phi}_{0 x}\right|_{0} ^{1} .
$$

Using (1.4) and (2.5), we estimate

$$
L_{\varepsilon} \tilde{\phi}_{0}=\varepsilon^{\mu+2} g^{\prime}(x) e^{-d / \varepsilon} \tilde{\phi}_{0 x} .
$$

Since $L_{\varepsilon} \tilde{\phi}_{0}$ is exponentially small and $\tilde{\phi}_{0}$ is of one sign, we have that $\phi_{0} \sim N_{0} \tilde{\phi}_{0}$ away from $O(\varepsilon)$ regions near the endpoints at $x=0$ and $x=1$ where $N_{0}$ is a normalization constant. However, this approximate form for $\phi_{0}$ does not satisfy the homogeneous 
boundary conditions in $(2.7 \mathrm{~b})$ and so we cannot use it to calculate $\phi_{0}(0)$ and $\phi_{0}(1)$. Instead, these quantities are calculated after constructing boundary layer profiles for $\phi_{0}$ near each endpoint.

Since $A$ is exponentially close to 1 , the boundary layer analysis given in [22] for the case $A \equiv 1$ can be used to calculate

$$
\phi_{0}(0) \sim 2 N_{0} a_{-} \nu_{-} e^{-\nu_{-} x_{0} / \varepsilon}, \quad \phi_{0}(1) \sim 2 N_{0} a_{+} \nu_{+} e^{-\nu_{+}\left(1-x_{0}\right) / \varepsilon} .
$$

Then, since the dominant contribution to the inner product integrals arises from the region near $x=x_{0}$, the left side of (2.8) is estimated as

$$
\left(\phi_{0}, \tilde{\phi}_{0}\right) \sim N_{0}\left(\tilde{\phi}_{0}, \tilde{\phi}_{0}\right) \sim \varepsilon \beta_{0} N_{0} ; \quad \beta_{0} \equiv \int_{-\infty}^{\infty}\left[u_{c}^{\prime}(z)\right]^{2} d z=\int_{s_{-}}^{s_{+}}[2 V(u)]^{1 / 2} d u .
$$

Next, we use (2.9) to estimate

$$
\left(\phi_{0}, L_{\varepsilon} \tilde{\phi}_{0}\right) \sim N_{0}\left(\tilde{\phi}_{0}, L_{\varepsilon} \tilde{\phi}_{0}\right) \sim N_{0} \varepsilon^{\mu+2} e^{-d / \varepsilon} \int_{-\infty}^{\infty} u_{c}^{\prime}(z) g^{\prime}\left(x_{0}+\varepsilon z\right) u_{c}^{\prime \prime}(z) d z .
$$

By using a Taylor series expansion for $g^{\prime}\left(x_{0}+\varepsilon z\right)$, we get

$$
\left(\phi_{0}, L_{\varepsilon} \tilde{\phi}_{0}\right) \sim-N_{0} \varepsilon^{\mu+2} e^{-d / \varepsilon} \sum_{k=0}^{\infty} \varepsilon^{k} g^{(k+1)}\left(x_{0}\right) \gamma_{k}
$$

where the coefficients $\gamma_{k}$ are defined by

$$
\gamma_{k} \equiv-\frac{1}{k !} \int_{-\infty}^{\infty} u_{c}^{\prime}(z) u_{c}^{\prime \prime}(z) z^{k} d z, \quad k=0,1, \ldots
$$

The first two coefficients are readily calculated to be

$$
\gamma_{0}=0, \quad \gamma_{1}=\frac{\beta_{0}}{2} .
$$

Moreover, if $u_{c}(z)$ is an even function, then $\gamma_{2 k}=0$. Finally, substituting (2.10)(2.13) into (2.8), we obtain the following key estimate for $\lambda_{0}$ :

Proposition 2.1 (Exponentially small eigenvalue). For $\varepsilon \rightarrow 0$, the exponentially small eigenvalue of (2.7) satisfies

$$
\begin{aligned}
\lambda_{0}=\lambda_{0}\left(x_{0}\right) \sim 2 \beta_{0}^{-1}\left\{a_{+}^{2} \nu_{+}^{3} e^{-2 \nu_{+}\left(1-x_{0}\right) / \varepsilon}\right. & \left.+a_{-}^{2} \nu_{-}^{3} e^{-2 \nu_{-} x_{0} / \varepsilon}\right\} \\
& -\beta_{0}^{-1} \varepsilon^{\mu+1} e^{-d / \varepsilon} \sum_{k=1}^{\infty} \varepsilon^{k} g^{(k+1)}\left(x_{0}\right) \gamma_{k} .
\end{aligned}
$$

Here $\nu_{ \pm}, a_{ \pm}$are defined in (2.3), $\beta_{0}$ is defined in (2.11), and $\gamma_{k}$ is defined in (2.14).

2.2. The metastabilty analysis. We now derive a differential equation for the location $x_{0}=x_{0}(t)$ of the internal layer trajectory. We first expand the solution $w$ to (2.5) in terms of the eigenfunctions $\phi_{j}$ of (2.7) as

$$
w(x, t)=\sum_{j=0}^{\infty} \frac{c_{j}(t)}{\lambda_{j}} \phi_{j}(x) .
$$


The coefficients $c_{j}$, which are found by integrating by parts, are

$$
c_{j}=-\varepsilon^{-1}\left(A x_{0}^{\prime} u_{c}^{\prime}, \phi_{j}\right)-\varepsilon\left(A_{x} u_{c}^{\prime}, \phi_{j}\right)-\left.\varepsilon^{2} A w_{x} \phi_{j}\right|_{0} ^{1}, \quad j=0,1, \ldots
$$

Since $\lambda_{0} \rightarrow 0$ as $\varepsilon \rightarrow 0$, a necessary condition for the solvability of (2.5) is that $c_{0} \rightarrow 0$ as $\varepsilon \rightarrow 0$. Setting $c_{0}=0$ in (2.18), we obtain the asymptotic differential equation for $x_{0}=x_{0}(t)$ :

$$
\varepsilon^{-1} x_{0}^{\prime}\left(A u_{c}^{\prime}, \phi_{0}\right) \sim-\varepsilon\left(A_{x} u_{c}^{\prime}, \phi_{0}\right)-\left.\varepsilon^{2} \phi_{0} A v_{x}\right|_{0} ^{1} .
$$

To obtain an explicit differential equation for $x_{0}(t)$, we must evaluate the inner product integrals and the boundary terms in (2.19). The dominant contributions to the inner product integrals arise from the region near $x=x_{0}$.

First, the boundary terms in (2.19) can be calculated asymptotically from (2.5) and (2.10) as

$$
\left.\varepsilon^{2} A \phi_{0} w_{x}\right|_{0} ^{1} \sim 2 N_{0} \varepsilon\left(-a_{+}^{2} \nu_{+}^{2} e^{-2 \nu_{+}\left(1-x_{0}\right) / \varepsilon}+a_{-}^{2} \nu_{-}^{2} e^{-2 \nu_{-} x_{0} / \varepsilon}\right) .
$$

Now, to evaluate $\left(A_{x} u_{c}^{\prime}, \phi_{0}\right)$, we use (1.4) and $\phi_{0} \sim N_{0} u_{c}^{\prime}$ to get

$$
\left(A_{x} u_{c}^{\prime}, \phi_{0}\right) \sim N_{0} \varepsilon^{\mu+1} e^{-d / \varepsilon} \int_{-\infty}^{\infty} g^{\prime}\left(x_{0}+\varepsilon z\right)\left[u_{c}^{\prime}(z)\right]^{2} d z
$$

By using a Taylor series expansion of $g\left(x_{0}+\varepsilon z\right)$, we obtain

$$
\left(A_{x} u_{c}^{\prime}, \phi_{0}\right) \sim N_{0} \varepsilon^{\mu+1} e^{-d / \varepsilon} \sum_{k=0}^{\infty} \varepsilon^{k} g^{(k+1)}\left(x_{0}\right) \beta_{k}
$$

where the coefficients $\beta_{k}$ are defined by

$$
\beta_{k}=\frac{1}{k !} \int_{-\infty}^{\infty}\left[u_{c}^{\prime}(z)\right]^{2} z^{k} d z, \quad k=0,1, \ldots
$$

Upon integrating by parts, we can show that $\beta_{k}=2 \gamma_{k+1}$ for $k \geq 0$ where $\gamma_{k}$ is defined in (2.14). Next, for $\varepsilon \rightarrow 0$, we estimate the left side of (2.19) to get

$$
\varepsilon^{-1} x_{0}^{\prime}\left(A u_{c}^{\prime}, \phi_{0}\right) \sim N_{0} \beta_{0} x_{0}^{\prime} .
$$

Finally, substituting (2.20), (2.22), and (2.24) into (2.19), we obtain our main result for the metastable dynamics associated with the generalized Ginzburg-Landau equation (1.1):

Proposition 2.2 (Metastable dynamics). For $\varepsilon \rightarrow 0$ and $t \gg 1$, a one-layer metastable pattern for (1.1) is represented by $u(x, t) \sim u_{c}\left[\varepsilon^{-1}\left(x-x_{0}(t)\right)\right]$ where the internal layer trajectory $x_{0}(t)$ satisfies the asymptotic differential equation

$$
\begin{aligned}
x_{0}^{\prime} \sim h\left(x_{0}\right) \equiv 2 \varepsilon \beta_{0}^{-1}\left[a_{+}^{2} \nu_{+}^{2} e^{-2 \nu_{+}\left(1-x_{0}\right) / \varepsilon}-a_{-}^{2} \nu_{-}^{2} e^{-2 \nu_{-} x_{0} / \varepsilon}\right] \\
-\varepsilon^{\mu+2} e^{-d / \varepsilon} \beta_{0}^{-1} \sum_{k=0}^{\infty} \varepsilon^{k} \beta_{k} g^{(k+1)}\left(x_{0}\right) .
\end{aligned}
$$


Here $\nu_{ \pm}, a_{ \pm}$are defined in (2.3), $\beta_{k}$ for $k \geq 0$ is defined in (2.23), and $u_{c}(z)$ is defined in (2.2).

The following equilibrium result is obtained by setting $x_{0}^{\prime}=0$ in (2.25):

Corollary 2.3 (Equilibrium). For $\varepsilon \rightarrow 0$, an equilibrium solution $U(x ; \varepsilon)$ to (1.1) corresponding to a one-layer pattern is given by $U(x ; \varepsilon) \sim u_{c}\left[\varepsilon^{-1}\left(x-x_{0}^{m}\right)\right]$ where $u_{c}(z)$ is defined in (2.2) and $x_{0}^{m}$ satisfies the nonlinear algebraic equation $h\left(x_{0}\right)=0$, i.e.,

$$
a_{+}^{2} \nu_{+}^{2} e^{-2 \nu_{+}\left(1-x_{0}\right) / \varepsilon}-a_{-}^{2} \nu_{-}^{2} e^{-2 \nu_{-} x_{0} / \varepsilon}=\frac{1}{2} \varepsilon^{\mu+1} e^{-d / \varepsilon} \sum_{k=0}^{\infty} \varepsilon^{k} \beta_{k} g^{(k+1)}\left(x_{0}\right)
$$

We now discuss the behavior of the equilibrium solutions for $x_{0}(t)$. We first observe that in (2.25), $h(0)<0$ and $h(1)>0$ for $\varepsilon \rightarrow 0$. Thus, there exists at least one equilibrium value $x_{0}^{m}$ for $x_{0}(t)$. The existence of any other equilibrium value for $x_{0}$ depends on the constants $d$ and $\mu$ and the function $g^{\prime}(x)$. For example, when $d>0$ is sufficiently large, the terms in (2.26) proportional to $e^{-d / \varepsilon}$ are insignificant and, consequently, the equilibrium value for $x_{0}$ is given uniquely by

$$
x_{0}^{m} \sim \frac{\nu_{+}}{\nu_{-}+\nu_{+}}-\frac{\varepsilon}{\nu_{-}+\nu_{+}} \log \left[\frac{a_{+} \nu_{+}}{a_{-} \nu_{-}}\right] .
$$

Alternatively, when $d>0$ is sufficiently small, the right side of (2.26) dominates the left side of (2.26) and, consequently, for $\varepsilon \rightarrow 0,(2.26)$ has a root $x_{0}^{m}$ near each zero of $g^{\prime}(x)$. As shown in the examples below, when $d$ is near some critical value so that the right and left sides of (2.26) balance as $\varepsilon \rightarrow 0$, we can have an equilibrium internal layer solution centered at a different point on the interval $[0,1]$.

Although the only stable equilibrium solutions to (1.3) in a convex domain are constants, the generalized G-L equation (1.1) may admit stable spatially dependent equilibrium solution with an internal layer structure. Let $x_{0}^{m}$ satisfy $h\left(x_{0}^{m}\right)=0$. Then, since $\beta_{k}=2 \gamma_{k+1}$, as seen by comparing (2.14) and (2.23), we can show that $h^{\prime}\left(x_{0}^{m}\right)=2 \lambda_{0}\left(x_{0}^{m}\right)$ where $\lambda_{0}$ is given in (2.16). This shows that the decay rate for the differential equation (2.25) associated with infinitesimal perturbations about $x_{0}^{m}$ is $2 \lambda_{0}^{m}$ where $\lambda_{0}^{m} \equiv \lambda_{0}\left(x_{0}^{m}\right)$. This leads to the following criterion for the stability of the equilibrium internal layer solutions:

Corollary 2.4 (Stability of equilibrium). Let $x_{0}^{m}$ satisfy $h\left(x_{0}^{m}\right)=0$. Then the equilibrium solution to (1.1) has the form $U \sim u_{c}\left[\left(x-x_{0}^{m}\right) / \varepsilon\right]$ and is stable (unstable) if $\lambda_{0}\left(x_{0}^{m}\right)<0\left(\lambda_{0}\left(x_{0}^{m}\right)>0\right)$. Here $u_{c}(z), \lambda_{0}\left(x_{0}\right)$, and $h\left(x_{0}\right)$ are given in (2.2), (2.16), and (2.25).

Using this corollary, it follows that an equilibrium solution with an internal layer located at $x_{0}=x_{0}^{m}$ is unstable when $g^{\prime \prime}\left(x_{0}^{m}\right)<0$. Since $g^{\prime \prime}(x)<0$ corresponds to a convex domain in higher dimensions, this result re-states the conclusion in [5] and [18] concerning the instability of non-constant steady-state solutions to (1.3) in convex domains. However, when $g^{\prime \prime}\left(x_{0}^{m}\right)>0$, then $\lambda_{0}^{m}$ can be negative for certain choices of $\mu$ and $d$, resulting in a stable internal layer solution centered at $x_{0}^{m}$. The key point to construct a stable equilibrium solution is to guarantee that (2.25) has multiple equilibria corresponding to simple zeroes of $h\left(x_{0}\right)$. Then, we must have exactly one stable equilibrium of (2.25) between every two consecutive unstable equilibria. We will see from the examples below that this can be realized by selecting the cross-sectional profile $A(x, \varepsilon)$ (i.e., $g(x))$ appropriately. 
2.3. Comparison of asymptotic and numerical results. We now compare the asymptotic results obtained above with the corresponding full numerical results computed directly from (1.1). We also show the existence of stable equilibrium solutions with an internal layer structure to the generalized G-L equation (1.1).

In all of the calculations below, we have taken $Q(u)=2\left(u-u^{3}\right)$, for which $a_{+}=a_{-}=2, \nu_{+}=\nu_{-}=2$, and $u_{s}(z)=\tanh (z)$. In addition, we calculate that $\beta_{0}=4 / 3, \gamma_{1}=2 / 3, \gamma_{3}=\left(\pi^{2}-6\right) / 36$, and $\gamma_{2 k}=0$ for $k \geq 0$. Thus, (2.16) becomes

$$
\begin{aligned}
\lambda_{0}=\lambda_{0}\left(x_{0}\right) \sim 48\left[e^{-4\left(1-x_{0}\right) / \varepsilon}+e^{-4 x_{0} / \varepsilon}\right] & \\
& \quad-\frac{1}{2} \varepsilon^{\mu+2} e^{-d / \varepsilon}\left[g^{\prime \prime}\left(x_{0}\right)+\frac{\pi^{2}-6}{24} g^{(4)}\left(x_{0}\right) \varepsilon^{2}+\cdots\right] .
\end{aligned}
$$

Noting that $\beta_{k}=2 \gamma_{k+1}$, the differential equation (2.25) becomes

$$
\begin{aligned}
x_{0}^{\prime} \sim h\left(x_{0}\right)=24 \varepsilon\left[e^{-4\left(1-x_{0}\right) / \varepsilon}-e^{-4 x_{0} / \varepsilon}\right] & \\
& -\varepsilon^{\mu+2} e^{-d / \varepsilon}\left[g^{\prime}\left(x_{0}\right)+\frac{\pi^{2}-6}{24} g^{(3)}\left(x_{0}\right) \varepsilon^{2}+\cdots\right] .
\end{aligned}
$$

To check the validity of (2.29), we solved (1.1) numerically for a number of choices of $g(x)$, three of which are described below.

To compute numerical solutions to (1.1), we use a transverse method of lines approach (cf. [2]). This method is based on replacing the time derivative in (1.1) by a difference approximation and then solving the resulting boundary value problems in space. More specifically, we convert the time-dependent problem (1.1) to a set of boundary value problems using the second order Backward Differential Formulas (BDF) [2], which we solve at each time step using the boundary value solver COLSYS [1]. Since the motion of the internal layer solutions is exponentially slow, we found it necessary to implement a time-stepping control strategy to efficiently track the solutions to (1.1) over long time intervals. To achieve this, we used the $l_{2}$-norm of the difference between the solutions of the second order and the third order BDF schemes as an error indicator to reject large inaccurate time steps or to enlarge unnecessary small time steps. See [21] for details of these algorithms, where they were used in a different context.

The metastablity result (2.29) is valid only after the completion of an $O(1)$ transient period that describes the formation of an internal layer from initial data. In the computations below, we took $u(x, 0)=u_{c}\left(\left[x-x_{0}^{0}\right] / \varepsilon\right)$ as the initial data for (1.1) where $u_{c}(z)$ is defined in $(2.2)$ and $x_{0}^{0} \in(0,1)$ is the initial zero of $u$. To eliminate any unwanted transient effects, we computed the solution to (1.1) with this initial data until $t=5$. At this time, $x_{0}^{0}$ is reset to be the zero of $u$ predicted by the numerical method. This new value for $x_{0}^{0}$ is used as the initial condition for (2.29). The differential equation (2.29) is then solved numerically for $x_{0}(t)$ using the initial value solver DP12 [3] and for $t\left(x_{0}\right)$ using a numerical quadrature, and the results are compared with corresponding numerical results for the zero of $u$ computed from the finite difference scheme.

EXAMPLE 2.1. Let $g(x)=-\frac{1}{2}\left(x-\frac{1}{2}\right)^{2}$, which corresponds to a convex domain. Then a solution to the equilibrium problem $h\left(x_{0}\right)=0$ for $(2.29)$ is $x_{0}^{m}=1 / 2$, independent of the constants $\mu$ and $d$. This is the only solution to $h\left(x_{0}\right)=0$, since $g^{\prime \prime}\left(x_{0}\right)<0$ implies that $h^{\prime}\left(x_{0}\right)>0$ for $x_{0} \in[0,1]$. This unique equilibrium solution 
TABLE 1. A comparison of the asymptotic and numerical results for $t=t\left(x_{0}\right)$ for $Q(u)=2\left(u-u^{3}\right)$ and $g(x)=-\frac{1}{2}\left(x-\frac{1}{2}\right)^{2}$ with $\varepsilon=0.05, \mu=0, d=0.4$, and $x_{0}(0)=0.4$.

\begin{tabular}{|c|c|c|}
\hline$x_{0}$ & $t$ (asymptotic) & $t$ (numerical) \\
\hline 0.3991489 & $0.100998498 \times 10^{5}$ & $0.100998673 \times 10^{5}$ \\
0.3902339 & $0.111103064 \times 10^{6}$ & $0.111104540 \times 10^{6}$ \\
0.3459655 & $0.515111092 \times 10^{6}$ & $0.515123233 \times 10^{6}$ \\
0.3013976 & $0.818109359 \times 10^{6}$ & $0.818137253 \times 10^{6}$ \\
0.2646857 & $0.102010258 \times 10^{7}$ & $0.102014660 \times 10^{7}$ \\
0.2024301 & $0.127991981 \times 10^{7}$ & $0.127995306 \times 10^{7}$ \\
0.1683312 & $0.133126841 \times 10^{7}$ & $0.133127901 \times 10^{7}$ \\
0.1350323 & $0.133755526 \times 10^{7}$ & $0.133756144 \times 10^{7}$ \\
0.0753443 & $0.133805967 \times 10^{7}$ & $0.133806497 \times 10^{7}$ \\
\hline
\end{tabular}

$x_{0}^{m}=1 / 2$ is unstable since $\lambda_{0}(1 / 2)>0$ in (2.28). This conclusion is confirmed by the full numerical results shown in Table 1. This table displays the asymptotic and numerical results for the elapsed time as a function of the internal layer location $x_{0}$ for $\varepsilon=0.05, \mu=0$, and $d=0.4$, when the initial location is $x_{0}(0)=0.4$. The results for $t=t\left(x_{0}\right)$ agree to at least four significant decimal places.

EXAMPLE 2.2. We choose $g(x)=\frac{1}{2}\left(x-\frac{1}{2}\right)^{2}$, which corresponds to a non-convex domain. Again, $x_{0}^{m}=1 / 2$ is an equilibrium solution to (2.29) for any $\mu$ and $d$. However, this solution can be stable depending on the values of $\mu$ and $d$. From (2.28), a simple calculation gives $\lambda_{0}(1 / 2)=-\frac{1}{2} \varepsilon^{\mu+2} e^{-d / \varepsilon}+96 e^{-2 / \varepsilon}$. Let $d_{c}$ be the zero of $\lambda_{0}(1 / 2)$ as a function of $d$, i.e., $d_{c}=2-\varepsilon \log 192+(\mu+2) \varepsilon \log \varepsilon$. Then, from Corollary 2.4, the equilibrium $x_{0}^{m}=1 / 2$ is stable (unstable) when $d<d_{c}$ $\left(d>d_{c}\right)$. Given $\mu=-2$ and $\varepsilon=0.1$, we have $d_{c} \approx 1.4742$. In Figure 2.3, we plot the numerical solution to (1.1) at different times for $d=1.4$ and $d=1.5$. For $d=1.5$ and $x_{0}(0)=0.49$, we observe that the internal layer located at $x_{0}(t)$ moves at an accelerating speed away from $x_{0}^{m}=\frac{1}{2}$ and eventually it collapses against the wall at $x=0$. Alternatively, for $d=1.4$ and $x_{0}(0)=0.45$ the layer drifts toward its equilibrium location at $x_{0}^{m}=\frac{1}{2}$ at an exceedingly slow rate. Thus, this example demonstrates the influence of the constants $\mu$ and $d$ on the stability of the equilibrium solution. Comparisons between the asymptotic and numerical results for the internal layer trajectories are displayed in Table 2a for $d=1.4$ and in Table $2 \mathrm{~b}$ for $d=1.5$. They agree to at least $3-4$ significant digits.

By plotting $h\left(x_{0}\right)$ in (2.29) versus $x_{0}$, we can show that $x_{0}^{m}=1 / 2$ is the only equilibrium to (2.29) when it is unstable. Alternatively, if the equilibrium $x_{0}^{m}=1 / 2$ is stable, then (2.29) has two additional (unstable) equilibria that emerge from a pitchfork bifurcation as $d$ is decreased below $d=d_{c}$. In particular, for the parameter values $\varepsilon=0.1, \mu=-2$, and $d=1.4$, we calculate that there are two other equilibria at $x_{0}^{m} \approx 0.4435$ and $x_{0}^{m} \approx 0.5565$. For a more general $g(x)$, the set of equilibria to (2.29) consists, for $\varepsilon \rightarrow 0$, of the zeroes of $g^{\prime}\left(x_{0}\right)$ and probably one or two others near the endpoints, provided that $d<2 \min \left(\nu_{-} x_{m}, \nu_{+}\left(1-x_{M}\right)\right)$ where $x_{m}$ and $x_{M}$ are the smallest and largest zeros of $g^{\prime}(x)$ on the interval $[0,1]$. Since the equilibrium solution closest to the endpoint $x=0$ or $x=1$ is unstable, a stable equilibrium $x_{0}^{m}$ must be near those zeros of $g^{\prime}(x)$ satisfying $g^{\prime \prime}\left(x_{0}^{m}\right)>0$. This analysis is illustrated in the next example. 
TABLE 2a. A comparison of the asymptotic and numerical internal layer trajectories for $Q(u)=2\left(u-u^{3}\right)$ and $g(x)=\frac{1}{2}\left(x-\frac{1}{2}\right)^{2}$ with $\varepsilon=0.1, \mu=-2$, $d=1.4$, and $x_{0}(0)=0.45$.

\begin{tabular}{|c|c|c|}
\hline$t$ & $x_{0}$ (asymptotic) & $x_{0}$ (numerical) \\
\hline $0.1009705 \times 10^{4}$ & 0.4500052 & 0.4500052 \\
$0.5824404 \times 10^{5}$ & 0.4503374 & 0.4503374 \\
$0.6115657 \times 10^{6}$ & 0.4541856 & 0.4541 .852 \\
$0.1266815 \times 10^{7}$ & 0.4600576 & 0.4600546 \\
$0.3232563 \times 10^{7}$ & 0.4790305 & 0.4790227 \\
$0.4510299 \times 10^{7}$ & 0.4875179 & 0.4875137 \\
$0.8933232 \times 10^{7}$ & 0.4981489 & 0.4981502 \\
$0.1586249 \times 10^{8}$ & 0.4999096 & 0.4999098 \\
$0.2243776 \times 10^{9}$ & 0.5 & 0.5 \\
\hline
\end{tabular}

TABLE 2b. A comparison of the asymptotic and numerical internal layer trajectories for $Q(u)=2\left(u-u^{3}\right)$ and $g(x)=\frac{1}{2}\left(x-\frac{1}{2}\right)^{2}$ with $\varepsilon=0.1, \mu=-2$, $d=1.5$, and $x_{0}(0)=0.49$.

\begin{tabular}{|c|c|c|}
\hline$x_{0}$ & $t$ (asymptotic) & $t$ (numerical) \\
\hline 0.4899742 & $0.255661494 \times 10^{5}$ & $0.255661329 \times 10^{5}$ \\
0.4892876 & $0.679551367 \times 10^{6}$ & $0.679517141 \times 10^{6}$ \\
0.4758890 & $0.747594011 \times 10^{7}$ & $0.747469530 \times 10^{7}$ \\
0.4519193 & $0.105770564 \times 10^{8}$ & $0.105738718 \times 10^{8}$ \\
0.4263499 & $0.113221195 \times 10^{8}$ & $0.113185051 \times 10^{8}$ \\
0.4015483 & $0.115273938 \times 10^{8}$ & $0.115236708 \times 10^{8}$ \\
0.3522899 & $0.116191551 \times 10^{8}$ & $0.116153855 \times 10^{8}$ \\
0.3018846 & $0.116312395 \times 10^{8}$ & $0.116274632 \times 10^{8}$ \\
0.2021744 & $0.116330380 \times 10^{8}$ & $0.116292599 \times 10^{8}$ \\
0.0794917 & $0.116330716 \times 10^{8}$ & $0.116292938 \times 10^{8}$ \\
\hline
\end{tabular}

EXAMPLE 2.3. We now consider $g(x)=\int_{0}^{x}\left(s-\frac{1}{3}\right)\left(s-\frac{2}{3}\right) d s$, which has one maximum at $x_{1}=\frac{1}{3}$ and one minimum at $x_{2}=\frac{2}{3}$. From the discussion before, since $g^{\prime \prime}\left(x_{1}\right)<0\left(g^{\prime \prime}\left(x_{2}\right)>0\right)$, we expect that when $d>0$ is sufficiently small, the equilibrium of (2.29) near $x_{1}\left(x_{2}\right)$ is unstable (stable). This is confirmed by the numerical results plotted in Figure 3 where $\varepsilon=0.08, \mu=0$, and $d=0.2$. Figure 3 a shows that the internal layer drifts slowly towards the stable equilibrium location at $x_{02}^{m} \approx 0.6608$ when its initial location is at $x_{0}(0)=0.4$. However, in Figure $3 \mathrm{~b}$, the internal layer with initial location $x_{0}(0)=0.333$ moves slowly towards the left and finally collapses against the wall at $x=0$. This shows that there is an unstable equilibrium near $x_{1}$, which is calculated from $(2.29)$ to be $x_{01}^{m} \approx 0.3401$. Corollaries 2.3 and 2.4 suggest that there is another unstable equilibrium $x_{03}^{m}$ between $x_{2}$ and the right endpoint. We compute from $(2.29)$ that $x_{0 \Xi}^{m} \approx 0.7762$. To confirm this conjecture, we compute the solution to (1.1) numerically for two different initial locations of the internal layer and we plot the corresponding numerical results at different times in Figure 4. From this figure, we observe that when $x_{0}(0)>x_{03}^{m}$ or $x_{02}^{m}<x_{0}(0)<x_{03}^{m}$, the internal layer moves exponentially slowly away from $x_{03}^{m}$ until it eventually collides with the endpoint $x=1$ or it reaches its stable equilibrium location at $x_{02}^{m}$, respectively. In summary, this example has three equilibrium internal layer solutions. The ones located at $x_{01}^{m}$ and $x_{03}^{m}$ are unstable, and the other one 


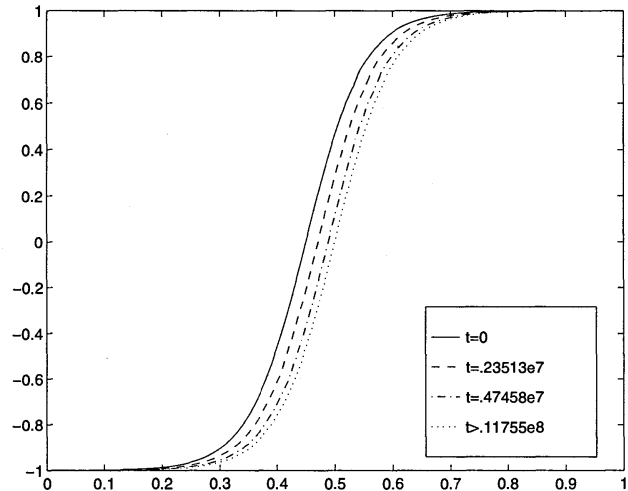

(a)

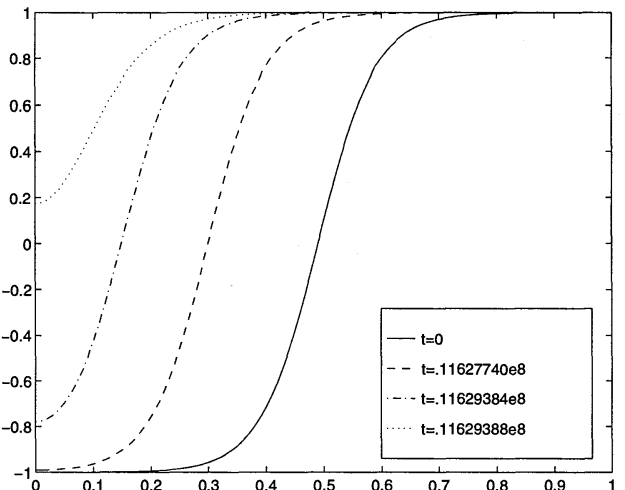

(b)

FIG. 2.1. Plots of the numerical solutions of (1.1) at different times with $Q(u)=2\left(u-u^{3}\right)$ and $g(x)=\frac{1}{2}\left(x-\frac{1}{2}\right)^{2}$ where $\varepsilon=0.1, \mu=-2$ with initial condition $u_{0}(x)=u_{c}\left(\left[x-x_{0}^{0}\right] / \varepsilon\right)$. (a) When $d=1.4$ and $x_{0}^{0}=0.45$, the internal layer moves towards its equilibrium $x_{0}^{m}=1 / 2$; (b) when $d=1.5$ and $x_{0}^{0}=0.49$, the internal layer moves towards the left and collides with $x=0$.

located at $x_{02}^{m}$ is stable.

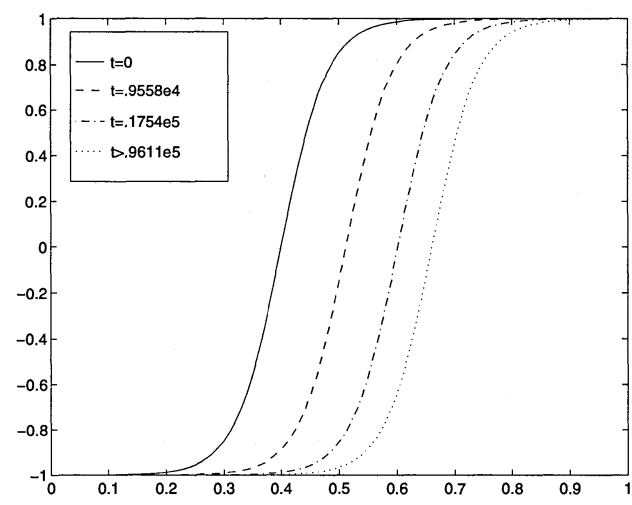

(a)

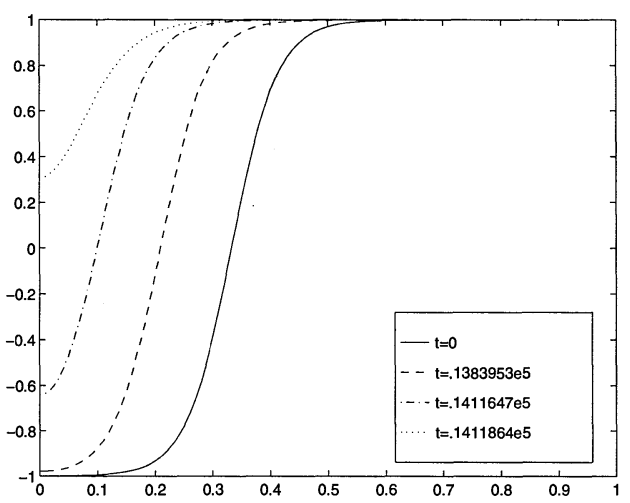

(b)

FIG. 2.2. Plots of the numerical solutions of (1.1) at different times with $Q(u)=2\left(u-u^{3}\right)$ and $g(x)=\int_{0}^{x}\left(s-\frac{1}{3}\right)\left(s-\frac{2}{3}\right) d s$ where $\varepsilon=0.08, \mu=0, d=0.2$ with initial condition $u_{0}(x)=u_{c}\left(\left[x-x_{0}^{0}\right] / \varepsilon\right)$. (a) When $x_{0}^{0}=0.4$, the internal layer moves towards $x_{02}^{m} \approx 0.6608$; (b) when $x_{0}^{0}=0.333$, the internal layer moves towards the left and collides with $x=0$.

For this example, in Tables $3 \mathrm{a}$ and $3 \mathrm{~b}$, we give a comparison between the asymptotic and numerical results for the evolution of the internal layers corresponding to Figure $3 \mathrm{a}$ and $3 \mathrm{~b}$, respectively. In these tables, the second column gives the numerical results for $x_{0}(t)$ or $t\left(x_{0}\right)$ while the third and fourth columns show the corresponding asymptotic results from (2.29) with the one term and the two term expansions for the second pair of brackets in (2.29), respectively. Since it may happen that $g^{\prime}\left(x_{0}\right)$ is close to zero during the evolution of an internal layer, the higher order term in (2.29) proportional to $g^{\prime \prime \prime}\left(x_{0}\right)$ can be quantitatively significant in some cases. In most cases, we find that the relative errors for the two-term expansion are below $0.002 \%$ in Table 
$3 \mathrm{a}$ and $0.02 \%$ in Table $3 \mathrm{~b}$, while they are only about $3 \%$ in Table $3 \mathrm{a}$ and $50 \%$ in Table $3 \mathrm{~b}$ for the one-term expansion. Thus, a two-term asymptotic expansion for (2.29) is certainly needed to obtain close quantitative agreement with the numerical results.

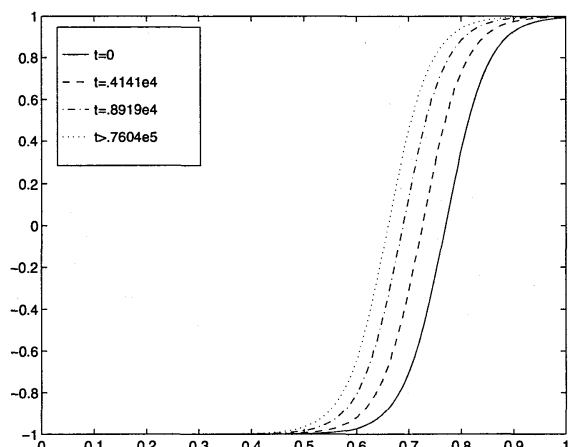

(a)

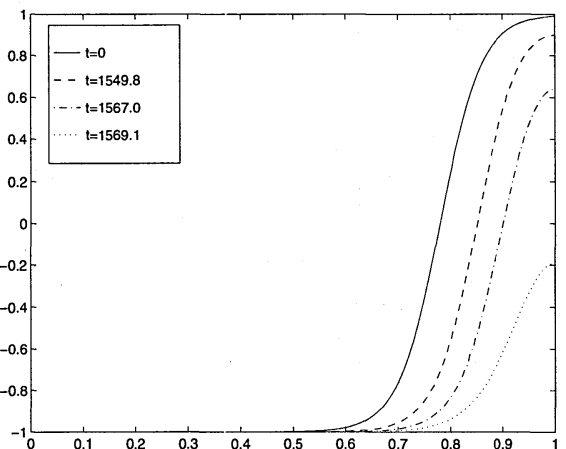

(b)

FIG. 2.3. Plots of the numerical solutions of (1.1) at different times with $Q(u)=2\left(u-u^{3}\right)$ and $g(x)=\int_{0}^{x}\left(s-\frac{1}{3}\right)\left(s-\frac{2}{3}\right) d s$ where $\varepsilon=0.08, \mu=0, d=0.2$ with initial condition $u_{0}(x)=u_{c}\left(\left[x-x_{0}^{0}\right] / \varepsilon\right)$. (a) When $x_{0}^{0}=0.76$, the internal layer moves towards $x_{02}^{m} \approx 0.6608$; (b) when $x_{0}^{0}=0.79$, the internal layer moves towards the right and collides with $x=1$.

TABLE 3a. A comparison of the asymptotic and numerical internal layer trajectories for $Q(u)=2\left(u-u^{3}\right)$ and $g(x)=\int_{0}^{x}(s-1 / 3)(s-2 / 3) d s$, with $\varepsilon=0.08, \mu=0$, $d=0.2$, and $x_{0}(0)=0.4$.

\begin{tabular}{|c|c|c|c|}
\hline$t$ & $x_{0}$ (numerical) & $x_{0}$ (asymptotic) 1-term & $x_{0}$ (asymptotic) 2-term \\
\hline $0.4531527 \times 10^{4}$ & 0.446231116 & 0.452053925 & 0.446234119 \\
$0.7487310 \times 10^{4}$ & 0.484045085 & 0.493797390 & 0.484052581 \\
$0.8965202 \times 10^{4}$ & 0.503953307 & 0.515317905 & 0.503963248 \\
$0.1339888 \times 10^{5}$ & 0.560669741 & 0.574424596 & 0.560683682 \\
$0.2881822 \times 10^{5}$ & 0.650108735 & 0.658569607 & 0.650107041 \\
$0.4544450 \times 10^{5}$ & 0.660137084 & 0.666792280 & 0.660135732 \\
$0.5741542 \times 10^{5}$ & 0.660748955 & 0.667250946 & 0.660748242 \\
$0.1175766 \times 10^{6}$ & 0.660847732 & 0.667319903 & 0.660847282 \\
$0.5677799 \times 10^{8}$ & 0.660847731 & 0.667319905 & 0.660847287 \\
\hline
\end{tabular}

We finally remark that, by taking $g(x)$ to be a periodic function, it is possible to construct a domain profile $A(x, \varepsilon)$ such that (1.1) has arbitrarily many (stable) equilibrium solutions.

3. A Burgers-like convection-diffusion-reaction equation. We now study (1.5) in the limit $\varepsilon \rightarrow 0$ with $c(x ; \varepsilon)$ as given in (1.7). The viscous shock solution for (1.5) can be approximated by

$$
u(x, t) \sim u_{s}\left[\varepsilon^{-1}\left(x-x_{0}(t)\right)\right]
$$

where the viscous shock profile $u_{s}(z)$ satisfies

$$
\begin{array}{cl}
u_{s}^{\prime}(z)=f\left[u_{s}(z)\right]-f\left(\alpha_{-}\right), & -\infty<z<\infty ; \quad u_{s}(0)=0 \\
u_{s}(z) \sim \alpha_{-}-a_{-} e^{\nu_{-} z}, \quad z \rightarrow-\infty ; & u_{s}(z) \sim \alpha_{+}+a_{+} e^{-\nu_{+} z}, \quad z \rightarrow+\infty
\end{array}
$$


TABLE 3b. A comparison of the asymptotic and numerical internal layer trajectories for $Q(u)=2\left(u-u^{3}\right)$ and $g(x)=\int_{0}^{x}(s-1 / 3)(s-2 / 3) d s$, with $\varepsilon=0.08, \mu=0$, $d=0.2$, and $x_{0}(0)=0.333$.

\begin{tabular}{|c|c|c|c|}
\hline$x_{0}$ & $t$ (numerical) & $t$ (asymptotic) 1 -term & $t$ (asymptotic) 2 -term \\
\hline 0.3327547 & $0.340885709 \times 10^{5}$ & $0.153239646 \times 10^{6}$ & $0.340916775 \times 10^{5}$ \\
0.3316803 & $0.147718828 \times 10^{6}$ & $0.444448389 \times 10^{6}$ & $0.147738247 \times 10^{6}$ \\
0.3058932 & $0.744277679 \times 10^{6}$ & $0.120475205 \times 10^{7}$ & $0.744387573 \times 10^{6}$ \\
0.2457738 & $0.101699029 \times 10^{7}$ & $0.148559051 \times 10^{7}$ & $0.101710846 \times 10^{7}$ \\
0.2015392 & $0.110200256 \times 10^{7}$ & $0.157152695 \times 10^{7}$ & $0.110210654 \times 10^{7}$ \\
0.1500169 & $0.116129605 \times 10^{7}$ & $0.163121386 \times 10^{7}$ & $0.116139950 \times 10^{7}$ \\
0.1145554 & $0.117455785 \times 10^{7}$ & $0.164451780 \times 10^{7}$ & $0.117466741 \times 10^{7}$ \\
0.1014824 & $0.117521955 \times 10^{7}$ & $0.164518040 \times 10^{7}$ & $0.117532979 \times 10^{7}$ \\
0.0747960 & $0.117546167 \times 10^{7}$ & $0.164542305 \times 10^{7}$ & $0.117557243 \times 10^{7}$ \\
\hline
\end{tabular}

The positive constants $\nu_{ \pm}$and $a_{ \pm}$are defined by

$$
\nu_{ \pm}=\mp f^{\prime}\left(\alpha_{ \pm}\right), \quad \log \left(\mp \frac{a_{ \pm}}{\alpha_{ \pm}}\right)=\mp \nu_{ \pm} \int_{0}^{\alpha_{ \pm}}\left[\frac{1}{f(s)-f\left(\alpha_{ \pm}\right)} \pm \frac{1}{\nu_{ \pm}\left(s-\alpha_{ \pm}\right)}\right] d s .
$$

We now look for a solution to (1.5) in the form

$$
u(x, t)=u_{s}\left[\varepsilon^{-1}\left(x-x_{0}(t)\right)\right]+v(x, t)
$$

where $v \ll u_{s}$ and $v_{t} \ll \partial_{t} u_{s}$. The trajectory $x_{0}=x_{0}(t)$ gives the approximate location of the zero of $u(x, t)$ during the metastable evolution. Substituting (3.4) into (1.5), and using (3.2), we obtain that $v$ satisfies the quasi-steady problem

$$
\begin{aligned}
\varepsilon v_{x x}-\left[f^{\prime}\left(u_{s}\right) v\right]_{x} & +\operatorname{ch}^{\prime}\left(u_{s}\right) v=-\operatorname{ch}\left(u_{s}\right)-\varepsilon^{-1} x_{0}^{\prime} u_{s}^{\prime}(z), \quad 0<x<1, \\
v(0, t) & =\alpha_{-}-u_{s}\left(-x_{0} / \varepsilon\right) \sim a_{-} e^{-\nu_{-} x_{0} / \varepsilon} \\
v(1, t) & =\alpha_{+}-u_{s}\left(\left[1-x_{0}\right] / \varepsilon\right) \sim-a_{+} e^{-\nu_{+}\left(1-x_{0}\right) / \varepsilon}
\end{aligned}
$$

where $z=\left(x-x_{0}(t)\right) / \varepsilon$.

As in [19], it is convenient to transform (3.5) to self-adjoint form by introducing a new variable $w(x, t)$ defined by

$$
v(x, t)=w(x, t) \psi(z), \quad \psi(z) \equiv\left[\frac{u_{s}^{\prime}(z)}{u_{s}^{\prime}(0)}\right]^{1 / 2}, \quad z=\varepsilon^{-1}\left(x-x_{0}\right) .
$$

Substituting (3.6) into (3.5), and using the asymptotic behavior of $\psi(z)$ as $z \rightarrow \pm \infty$, we find that $w(x, t)$ satisfies

$$
\begin{aligned}
L_{\varepsilon} w \equiv \varepsilon^{2} w_{x x}-V[x ; \varepsilon] w & \sim-\varepsilon \psi^{-1}\left(\operatorname{ch}\left(u_{s}\right)+\varepsilon^{-1} x_{0}^{\prime} u_{s}^{\prime}(z)\right), \quad 0<x<1 \\
w(0, t) & \sim\left[\frac{a_{-} f\left(\alpha_{-}\right)}{\nu_{-}}\right]^{1 / 2} e^{-\nu_{-} x_{0} / 2 \varepsilon} \\
w(1, t) & \sim-\left[\frac{a_{+} f\left(\alpha_{+}\right)}{\nu_{+}}\right]^{1 / 2} e^{-\nu_{+}\left(1-x_{0}\right) / 2 \varepsilon}
\end{aligned}
$$


Here $V(x ; \varepsilon)$ is defined by

$$
V(x ; \varepsilon) \equiv \frac{1}{4}\left[f^{\prime}\left(u_{s}(z)\right)\right]^{2}+\frac{1}{2} f^{\prime \prime}\left[u_{s}(z)\right] u_{s}^{\prime}(z)-\varepsilon \operatorname{ch}^{\prime}\left[u_{s}(z)\right]
$$

where $z=\varepsilon^{-1}\left(x-x_{0}\right)$ and $c=c(x ; \varepsilon)$ is given in (1.7).

3.1. The eigenvalue analysis. For a fixed $x_{0} \in(0,1)$, we now study the eigenvalue problem

$$
\begin{aligned}
L_{\varepsilon} \phi & =\lambda \phi, \quad 0<x<1, \\
\phi(0) & =\phi(1)=0, \quad(\phi, \phi)=1 .
\end{aligned}
$$

Here $(u, v) \equiv \int_{0}^{1} u v d x$. For this eigenproblem, the eigenvalues $\lambda_{j}$ for $j \geq 0$ are real and the principal eigenvalue $\lambda_{0}$ is exponentially small as $\varepsilon \rightarrow 0$. We now extend the analysis of [19] to give an estimate for $\lambda_{0}$ and for the corresponding eigenfunction $\phi_{0}$.

We first define the trial function $\tilde{\phi}_{0}$ by $\tilde{\phi}_{0}(x) \equiv \psi^{-1}(z) u_{s}^{\prime}(z)$ where $z=\left(x-x_{0}\right) / \varepsilon$ and $\psi$ is defined in (3.6). Then, applying Green's identity to $\phi_{0}$ and $\tilde{\phi}_{0}$, and using (1.7), we get

$$
\lambda_{0}\left(\phi_{0}, \tilde{\phi}_{0}\right)=\left(\phi_{0}, L_{\varepsilon} \tilde{\phi}_{0}\right)+\left.\varepsilon^{2} \phi_{0 x} \tilde{\phi}_{0}\right|_{0} ^{1}
$$

where

$$
L_{\varepsilon} \tilde{\phi}_{0}=-\varepsilon^{\mu+1} g^{\prime}(x) e^{-d / \varepsilon} h^{\prime}\left[u_{s}(z)\right] u_{s}^{\prime}(z) / \psi(z) \text {. }
$$

Since $L_{\varepsilon} \tilde{\phi}_{0}$ is exponentially small and $\tilde{\phi}_{0}$ is of one sign, we have that $\phi_{0} \sim N_{0} \tilde{\phi}_{0}$, except near the endpoints at $x=0$ and $x=1$. Here $N_{0}$ is a normalization constant. We must modify $\tilde{\phi}_{0}$ by inserting boundary layer profiles near the endpoints in order to satisfy the boundary conditions in $(3.9 \mathrm{~b})$. These boundary layers can be analyzed in the same way as in [19] and, from this analysis, we obtain that

$$
\begin{aligned}
& \phi_{0 x}(0) \sim-\varepsilon^{-1} N_{0} \nu_{-}\left[a_{-} \nu_{-} f\left(\alpha_{-}\right)\right]^{1 / 2} e^{-\nu_{-} x_{0} / 2 \varepsilon}, \\
& \phi_{0 x}(1) \sim \varepsilon^{-1} N_{0} \nu_{+}\left[a_{+} \nu_{+} f\left(\alpha_{+}\right)\right]^{1 / 2} e^{-\nu_{+}\left(1-x_{0}\right) / 2 \varepsilon} .
\end{aligned}
$$

Since the dominant contribution to each of the inner product integrals in (3.10a) arises from the region near $x=x_{0}$, we can calculate $\left(\phi_{0}, \tilde{\phi}_{0}\right)$ and $\left(\phi_{0}, L_{\varepsilon} \tilde{\phi}_{0}\right)$ using Laplace's method. As in [19], we estimate

$$
\left(\phi_{0}, \tilde{\phi}_{0}\right) \sim N_{0}\left(\tilde{\phi}_{0}, \tilde{\phi}_{0}\right) \sim \varepsilon N_{0} \int_{-\infty}^{\infty}\left[u_{s}^{\prime}(z)\right]^{2} \psi^{-2}(z) d z=\varepsilon N_{0}\left(\alpha_{-}-\alpha_{+}\right) f\left(\alpha_{-}\right) .
$$

To calculate $\left(\phi_{0}, L_{\varepsilon} \tilde{\phi}_{0}\right)$, we use $\left(\phi_{0}, L_{\varepsilon} \tilde{\phi}_{0}\right) \sim N_{0}\left(\tilde{\phi}_{0}, L_{\varepsilon} \tilde{\phi}_{0}\right)$. Substituting $\tilde{\phi}_{0}=\psi^{-1}(z)$ $u_{s}^{\prime}(z)$ into this expression, and using (3.6) and $u_{s}^{\prime}(0)=-f\left(\alpha_{-}\right)$, we derive

$$
\left(\phi_{0}, L_{\varepsilon} \tilde{\phi}_{0}\right) \sim N_{0} f\left(\alpha_{-}\right) \varepsilon^{\mu+2} e^{-d / \varepsilon} \int_{-\infty}^{\infty} g^{\prime}\left(x_{0}+\varepsilon z\right) u_{s}^{\prime}(z) h^{\prime}\left[u_{s}(z)\right] d z .
$$

A Taylor series expansion for $g^{\prime}\left(x_{0}+\varepsilon z\right)$ then yields

$$
\left(\phi_{0}, L_{\varepsilon} \tilde{\phi}_{0}\right) \sim-N_{0} f\left(\alpha_{-}\right) \varepsilon^{\mu+2} e^{-d / \varepsilon} \sum_{k=0}^{\infty} \varepsilon^{k} \gamma_{k} g^{(k+1)}\left(x_{0}\right)
$$


where the coefficients $\gamma_{k}$ in (3.14) are defined by

$$
\gamma_{k} \equiv-\frac{1}{k !} \int_{-\infty}^{\infty} u_{s}^{\prime}(z) h^{\prime}\left[u_{s}(z)\right] z^{k} d z, \quad k=0,1, \ldots
$$

Since we have assumed that $h^{\prime}(u)$ is bounded on the interval $\left[\alpha_{+}, \alpha_{-}\right]$, the exponential decay of $u_{s}^{\prime}(z)$ as $z \rightarrow \pm \infty$ ensures that $\gamma_{k}$ is finite for each $k \geq 0$. We can calculate $\gamma_{0}$ explicitly to get

$$
\gamma_{0}=h\left(\alpha_{-}\right)-h\left(\alpha_{+}\right)
$$

Notice that if $f(u)$ is even and $h(u)$ is odd, we get $\gamma_{2 k+1}=0$ for $k \geq 0$. Finally, substituting (3.11), (3.12), and (3.14) into (3.10a), we obtain the following key estimate for $\lambda_{0}$ :

Proposition 3.1 (Exponentially small eigenvalue). For $\varepsilon \rightarrow 0$, the exponentially small eigenvalue of (3.9) satisfies

$$
\begin{aligned}
\lambda_{0}=\lambda_{0}\left(x_{0}\right) \sim \frac{-1}{\alpha_{-}-\alpha_{+}}\left[a_{+} \nu_{+}^{2} e^{-\nu_{+}\left(1-x_{0}\right) / \varepsilon}\right. & \left.+a_{-} \nu_{-}^{2} e^{-\nu_{-} x_{0} / \varepsilon}\right] \\
& -\frac{\varepsilon^{\mu+1} e^{-d / \varepsilon}}{\alpha_{-}-\alpha_{+}} \sum_{k=0}^{\infty} \varepsilon^{k} \gamma_{k} g^{(k+1)}\left(x_{0}\right) .
\end{aligned}
$$

Here $\nu_{ \pm}, a_{ \pm}$are defined in (3.3), and $\gamma_{k}$ is defined in (3.15).

3.2. The metastability analysis. We now derive a differential equation for the location $x_{0}=x_{0}(t)$ of the internal layer trajectory. We first expand the solution $w$ to (3.7) in terms of the eigenfunctions $\phi_{j}$ of (3.9) as

$$
w(x, t)=\sum_{j=0}^{\infty} \frac{r_{j}(t)}{\lambda_{j}} \phi_{j}(x) .
$$

The coefficients $r_{j}$, which are found by integrating by parts, are

$$
r_{j}=-x_{0}^{\prime}\left(\phi_{j}, \psi^{-1} u_{s}^{\prime}\right)-\varepsilon\left(\phi_{j}, \psi^{-1} c h\right)+\left.\varepsilon^{2} w \phi_{j x}\right|_{0} ^{1}
$$

where $\psi$ is defined in (3.6). Since $\lambda_{0} \rightarrow 0$ as $\varepsilon \rightarrow 0$, a necessary condition for the solvability of (3.7) is that $r_{0} \rightarrow 0$ as $\varepsilon \rightarrow 0$. Setting $r_{0}=0$ in (3.19), we obtain the asymptotic differential equation for $x_{0}=x_{0}(t)$

$$
x_{0}^{\prime}\left(\phi_{0}, \psi^{-1} u_{s}^{\prime}\right) \sim-\varepsilon\left(\phi_{0}, \psi^{-1} c h\right)+\left.\varepsilon^{2} w \phi_{0 x}\right|_{0} ^{1} .
$$

To obtain an explicit differential equation for $x_{0}(t)$, we must evaluate the inner product integrals and the boundary terms in (3.20). The dominant contributions to the inner product integrals arise from the region near $x=x_{0}$.

First, we use (3.7b) $(3.7 \mathrm{c})$, and (3.11) to asymptotically calculate the last term on the right side of $(3.20)$ as

$$
\left.\varepsilon^{2} w \phi_{0 x}\right|_{0} ^{1} \sim N_{0} \varepsilon f\left(\alpha_{-}\right)\left\{a_{-} \nu_{-} e^{-\nu_{-} x_{0} / \varepsilon}-a_{+} \nu_{+} e^{-\nu_{+}\left(1-x_{0}\right) / \varepsilon}\right\} .
$$


Next, we evaluate the term on the left side of (3.20) as

$$
\left(\phi_{0}, \psi^{-1} u_{s}^{\prime}\right) \sim \varepsilon N_{0}\left(\alpha_{-}-\alpha_{+}\right) f\left(\alpha_{-}\right),
$$

which is the same as (3.12). To evaluate the first term on the right-hand side of (3.20), we use $\phi_{0} \sim N_{0} \tilde{\phi}_{0}$ and $\tilde{\phi}_{0}=\psi^{-1}(z) u_{s}^{\prime}(z)$ to get

$$
\varepsilon\left(\phi_{0}, \psi^{-1} c h\right) \sim N_{0} \varepsilon^{\mu+1} e^{-d / \varepsilon} f\left(\alpha_{-}\right) \int_{0}^{1} g^{\prime}(x) h\left(u_{s}\left[\left(x-x_{0}\right) / \varepsilon\right]\right) d x .
$$

Since $h\left(u_{s}(z)\right) \rightarrow h\left(\alpha_{ \pm}\right)$exponentially as $z \rightarrow \pm \infty$, we can evaluate the integral in (3.23) by decomposing it as

$$
\begin{aligned}
& \int_{0}^{1} g^{\prime}(x) h\left(u_{s}\left[\left(x-x_{0}\right) / \varepsilon\right]\right) d x \sim \int_{0}^{x_{0}}\left[h\left(u_{s}\right)-h\left(\alpha_{-}\right)\right] g^{\prime}(x) d x \\
+ & \int_{x_{0}}^{1}\left[h\left(u_{s}\right)-h\left(\alpha_{+}\right)\right] g^{\prime}(x) d x+h\left(\alpha_{-}\right)\left[g\left(x_{0}\right)-g(0)\right]+h\left(\alpha_{+}\right)\left[g(1)-g\left(x_{0}\right)\right] .
\end{aligned}
$$

The integrands in the two integrals on the right side of (3.24) are localized near $x=x_{0}$ and can be evaluated using a Taylor expansion to get

$$
\begin{aligned}
\int_{0}^{1} g^{\prime}(x) h\left(u_{s}\left[\left(x-x_{0}\right) / \varepsilon\right]\right) d x & \sim h\left(\alpha_{-}\right)\left[g\left(x_{0}\right)-g(0)\right] \\
& +h\left(\alpha_{+}\right)\left[g(1)-g\left(x_{0}\right)\right]+\varepsilon \sum_{k=0}^{\infty} \varepsilon^{k} \beta_{k} g^{(k+1)}\left(x_{0}\right)
\end{aligned}
$$

where the coefficients $\beta_{k}$, for $k=0,1, \ldots$, are defined by

$$
\beta_{k}=\frac{1}{k !} \int_{-\infty}^{0}\left(h\left[u_{s}(z)\right]-h\left(\alpha_{-}\right)\right) z^{k} d z+\frac{1}{k !} \int_{0}^{\infty}\left(h\left[u_{s}(z)\right]-h\left(\alpha_{+}\right)\right) z^{k} d z
$$

Using integrating by parts, it is readily seen that $\beta_{k}=\gamma_{k+1}$ where $\gamma_{k}$ was defined previously in (3.15). We also observe that when $f(u)$ is even and $h(u)$ is odd, then $\beta_{2 k}=0$ for $k \geq 0$.

Finally, substituting (3.21), (3.22), (3.25a) into (3.20), we obtain our main result for the metastable dynamics associated with (1.5):

Proposition 3.2 (Metastable dynamics). For $\varepsilon \rightarrow 0$ and $t \gg 1$, the metastable viscous shock dynamics for $(1.5)$ is represented by $u(x, t) \sim u_{s}\left[\varepsilon^{-1}\left(x-x_{0}(t)\right)\right]$ where the internal layer trajectory $x_{0}(t)$ satisfies the asymptotic differential equation

$$
\begin{aligned}
x_{0}^{\prime} \sim & M\left(x_{0}\right) \equiv \frac{1}{\alpha_{-}-\alpha_{+}}\left\{a_{-} \nu_{-} e^{-\nu_{-} x_{0} / \varepsilon}-a_{+} \nu_{+} e^{-\nu_{+}\left(1-x_{0}\right) / \varepsilon}\right\}-\frac{1}{\alpha_{-}-\alpha_{+}} \varepsilon^{\mu} e^{-d / \varepsilon} \\
& \times\left\{h\left(\alpha_{-}\right)\left[g\left(x_{0}\right)-g(0)\right]+h\left(\alpha_{+}\right)\left[g(1)-g\left(x_{0}\right)\right]+\varepsilon \sum_{k=0}^{\infty} \varepsilon^{k} \beta_{k} g^{(k+1)}\left(x_{0}\right)\right\} .
\end{aligned}
$$

Here the coefficients $a_{ \pm}$and $\nu_{ \pm}$are defined in (3.3), $\beta_{k}$ for $k \geq 0$ is defined in $(3.25 b)$, and $u_{s}(z)$ is defined in (3.2). 
The following equilibrium result is obtained by setting $x_{0}^{\prime}=0$ in (3.26):

COROllary 3.3 (Equilibrium). For $\varepsilon \rightarrow 0$, an equilibrium shock-layer solution to (1.5) is given asymptotically by $U \sim u_{s}\left[\left(x-x_{0}^{m}\right) / \varepsilon\right]$ where $u_{s}(z)$ is defined in (3.2) and $x_{0}=x_{0}^{m}$ satisfies the nonlinear algebraic equation $M\left(x_{0}\right)=0$, i.e.,

$$
\begin{aligned}
& a_{-} \nu_{-} e^{-\nu_{-} x_{0} / \varepsilon}-a_{+} \nu_{+} e^{-\nu_{+}\left(1-x_{0}\right) / \varepsilon}=\varepsilon^{\mu} e^{-d / \varepsilon}\left\{h\left(\alpha_{-}\right)\left[g\left(x_{0}\right)-g(0)\right]\right. \\
&\left.+h\left(\alpha_{+}\right)\left[g(1)-g\left(x_{0}\right)\right]+\varepsilon \sum_{k=0}^{\infty} \varepsilon^{k} \beta_{k} g^{(k+1)}\left(x_{0}\right)\right\} .
\end{aligned}
$$

To qualitatively understand the equilibrium problem for $x_{0}^{\prime}=M\left(x_{0}\right)$, we first note that $M(0)>0$ and $M(1)<0$ as $\varepsilon \rightarrow 0$ when $d>0$. Thus, there is at least one equilibrium solution for (3.26) as $\varepsilon \rightarrow 0$. If $d>0$ is sufficiently large, then the term in (3.26) proportional to $e^{-\varepsilon^{-1} d}$ can be neglected and hence the unique root $x_{0}^{m}$ of $M\left(x_{0}\right)=0$ is given asymptotically by

$$
x_{0}^{m} \sim \frac{\nu_{+}}{\nu_{-}+\nu_{+}}-\frac{\varepsilon}{\nu_{-}+\nu_{+}} \log \left(\frac{a_{+} \nu_{+}}{a_{-} \nu_{-}}\right) .
$$

Alternatively, if $d>0$ is sufficiently small, then $M\left(x_{0}\right)=0$ may have multiple roots for some choices of $g(x)$. This will be illustrated below for some specific examples.

Since $\gamma_{k}=\beta_{k-1}$, it follows that $M^{\prime}\left(x_{0}\right)=\lambda_{0}\left(x_{0}\right) / \varepsilon$. Thus, the decay rate associated with infinitesimal perturbations about $x_{0}^{m}$ is $\lambda_{0}\left(x_{0}^{m}\right) / \varepsilon$. This leads to the following criterion for the stability of the equilibrium shock-layer solution.

Corollary 3.4 (Stability of equilibrium). Let $x_{0}^{m}$ satisfy $M\left(x_{0}^{m}\right)=0$. Then the equilibrium solution to (1.5) has the form $U \sim u_{s}\left[\left(x-x_{0}^{m}\right) / \varepsilon\right]$ and is stable (unstable) if $\lambda_{0}\left(x_{0}^{m}\right)<0\left(\lambda_{0}\left(x_{0}^{m}\right)>0\right)$. Here $u_{s}(z), \lambda_{0}\left(x_{0}\right)$, and $M\left(x_{0}\right)$ are given in (3.2), (3.17), and (3.26). is that

It is easy to show that a sufficient condition for $M^{\prime}\left(x_{0}\right)<0$ on $x_{0} \in[0,1]$ as $\varepsilon \rightarrow 0$

$$
g^{\prime}\left(x_{0}\right)\left[h\left(\alpha_{-}\right)-h\left(\alpha_{+}\right)\right]>0, \quad \text { for all } x_{0} \in[0,1] .
$$

When this condition holds, the shock-layer solution centered at $x_{0}=x_{0}^{m}$ where $x_{0}^{m}$ is the unique root of $M\left(x_{0}\right)=0$, is stable for $\varepsilon \ll 1$. In particular, (3.29) is satisfied when $h^{\prime}(u)>0$ for $u \in\left[\alpha_{+}, \alpha_{-}\right]$and $g^{\prime}(x)>0$ on $x \in[0,1]$, which models a diverging nozzle flow as discussed following (1.7) above. More generally, a sufficient condition for the stability of a root $x_{0}^{m}$ of $M\left(x_{0}\right)=0$ is that (3.29) holds at $x_{0}=x_{0}^{m}$. This stability conclusion for an internal layer solution is consistent with [11].

However, when $g^{\prime}\left(x_{0}\right)\left[h\left(\alpha_{-}\right)-h\left(\alpha_{+}\right)\right]<0$ on $[0,1]$, the stability of an equilibrium internal layer solution can only be determined by explicitly calculating the sign of $\lambda_{0}\left(x_{0}^{m}\right)$ where $x_{0}^{m}$ is a root of $M\left(x_{0}\right)=0$. In this case, multiple equilibrium solutions for $x_{0}^{\prime}=M\left(x_{0}\right)$ are possible (see the examples below). We now illustrate the existence of multiple equilibria in this case when $d>0$ is sufficiently small and $\varepsilon \rightarrow 0$. Let's suppose that $g^{\prime}\left(x_{0}\right)<0$ on $[0,1]$ and $h\left(\alpha_{-}\right)>h\left(\alpha_{+}\right)>0$. Then, when $d>0$ is sufficiently small and $\varepsilon \rightarrow 0$, there is a root $x_{02}^{m}$ of $M\left(x_{0}\right)=0$ that is $O(\varepsilon)$ close to the unique solution of

$$
h\left(\alpha_{-}\right)\left[g\left(x_{0}\right)-g(0)\right]+h\left(\alpha_{+}\right)\left[g(1)-g\left(x_{0}\right)\right]=0 .
$$


The assumption $g^{\prime}\left(x_{02}^{m}\right)\left[h\left(\alpha_{-}\right)-h\left(\alpha_{+}\right)\right]<0$ then yields that $M^{\prime}\left(x_{02}^{m}\right)>0$ when $d>0$ is sufficiently small and $\varepsilon \rightarrow 0$. Hence, this root is unstable. However, when $d>0$ and $\varepsilon \rightarrow 0$, we calculate that $M(0)>0, M(1)<0, M^{\prime}(0)<0, M^{\prime}(1)<0$. Hence, there must exist additional roots $x_{01}^{m}$ and $x_{03}^{m}$ to $M\left(x_{0}\right)=0$ that satisfy $0<x_{01}^{m}<x_{02}^{m}$ and $x_{02}^{m}<x_{03}^{m}<1$ for which $M^{\prime}\left(x_{01}^{m}\right)<0$ and $M^{\prime}\left(x_{03}^{m}\right)<0$. Thus, these additional roots are stable equilibria of $x_{0}^{\prime}=M\left(x_{0}\right)$ and the profiles $u_{s}\left[\varepsilon^{-1}\left(x-x_{01}^{m}\right)\right]$ and $u_{s}\left[\varepsilon^{-1}\left(x-x_{03}^{m}\right)\right]$ correspond to stable internal layer solutions. Notice, as $d \rightarrow 0^{+}$, $x_{01}^{m} \rightarrow 0$ and $x_{03}^{m} \rightarrow 1$ so that these internal layer solutions become stable boundary layer solutions in agreement with the analysis in [11] for the case $d=0$.

3.3. Comparison of asymptotic and numerical results. We now compare the asymptotic results obtained above with the corresponding full numerical results computed directly from (1.5). For all of the calculations below, we consider the case

$$
f(u)=\frac{u^{2}}{2}, \quad h(u)=u, \quad \alpha_{-}=-\alpha_{+}=\alpha .
$$

As discussed in [20], and following (1.7) above, this problem models the transonic flow through a nozzle of cross-sectional area $A(x ; \varepsilon)=1+\varepsilon^{\mu} e^{-\varepsilon^{-1} d} g(x)$. We will consider nozzles of different cross-sectional areas by varying the term $g(x)$ in (3.17), (3.26), and (3.27). Recall that the nozzle is said to be divergent if $g^{\prime}(x)>0$ for all $x$, convergent if $g^{\prime}(x)<0$ for all $x$, and convergent-divergent if $g^{\prime}(x)$ has no definite sign. We use a similar numerical method as described in $\S 2.3$ above to compute numerical solutions to (1.5) and to compare with the corresponding asymptotic results.

When $f(u)=u^{2} / 2$ and $\alpha_{-}=-\alpha_{+}=\alpha$, we have $a_{ \pm}=2 \alpha \nu_{ \pm}=\alpha$ and $u_{s}(z)=$ $-\alpha \tanh (\alpha z / 2)$. We also calculate that $\left(\gamma_{0}, \gamma_{1}, \gamma_{2}, \gamma_{3}, \ldots\right)=\left(2 \alpha, 0, \pi^{2} / 3 \alpha, 0, \ldots\right)$. From (3.17), the principal eigenvalue $\lambda_{0}\left(x_{0}\right)$ satisfies

$$
\lambda_{0} \sim-\alpha^{2}\left(e^{-\alpha\left(1-x_{0}\right) / \varepsilon}+e^{-\alpha x_{0} / \varepsilon}\right)-\varepsilon^{\mu+1} e^{-d / \varepsilon}\left[g^{\prime}\left(x_{0}\right)+\frac{\pi^{2} \varepsilon^{2}}{6 \alpha^{2}} g^{\prime \prime \prime}\left(x_{0}\right)+\cdots\right] .
$$

In addition, since $\beta_{k}=\gamma_{k+1}$ for $k \geq 0,(3.26)$ becomes

$$
\begin{aligned}
x_{0}^{\prime} \sim M\left(x_{0}\right)=\alpha\left(e^{-\alpha x_{0} / \varepsilon}\right. & \left.-e^{-\alpha\left(1-x_{0}\right) / \varepsilon}\right)-\varepsilon^{\mu} e^{-d / \varepsilon} \\
& \times\left[\left(g\left(x_{0}\right)-\frac{g(0)+g(1)}{2}\right)+\frac{\pi^{2} \varepsilon^{2}}{6 \alpha^{2}} g^{\prime \prime}\left(x_{0}\right)+\cdots\right] .
\end{aligned}
$$

In most cases, the higher order terms in the square brackets on the right sides of (3.32) and (3.33) make only very minor improvements to the results. Thus, except when specified otherwise, they are ignored when making the comparisons below.

EXAMPLE 3.1. We first consider a divergent nozzle where $g(x)=C x$ for some $C \geq 0$. From (3.32) and (3.33), the only equilibrium value for $x_{0}(t)$ is $x_{0}^{m}=\frac{1}{2}$ and the principal eigenvalue $\lambda_{0}(1 / 2)$ is always negative for any $\mu$ and $d>0, C \geq 0$. Thus, from Corollary 3.3 and 3.4, there is a unique shock-layer solution centered at $x=1 / 2$ and it is stable. This agrees with the conclusion in [7] and [17] that flows along a divergent nozzle are always stable. The case $C=0$ was well-studied in [13], [15], and [19] and comparisons between asymptotic and numerical results can be found in [15] and [19].

EXAMPLE 3.2. We next consider a convergent nozzle where $g(x)=C x$ for some $C<0$. In this case, $x_{0}^{m}=1 / 2$ is still a root of $M\left(x_{0}\right)=0$ in (3.33), but now from 
(3.32) we calculate $\lambda_{0}(1 / 2)$ as

$$
\lambda_{0}(1 / 2) \sim-2 \alpha^{2} \varepsilon^{-1} e^{-\alpha / 2 \varepsilon}-\varepsilon^{\mu} e^{-d / \varepsilon} C .
$$

Thus, the stability of $x_{0}^{m}=1 / 2$ is determined by the values of $\mu, d$, and $C$. For example, if $\mu=-1$ and $d=\alpha / 2$, then the shock layer located at $x_{0}^{m}=\frac{1}{2}$ is stable (unstable) if $C>-2 \alpha^{2}\left(C<-2 \alpha^{2}\right)$. If $\mu=-1$ and $C=-2 \alpha^{2}$, then it is stable (unstable) if $d>\alpha / 2(d<\alpha / 2)$. These stability results are fully confirmed by the numerical results displayed in Tables 4 and 5, where $\alpha=1$. In these tables, we give the asymptotic and numerical results for $x_{0}(t)$ in the second and third columns respectively, and the error representing the difference between the asymptotic and numerical results in the fourth column. The asymptotic results agree with the numerical ones to at least five decimal places. From these tables, we observe that when the equilibrium $x_{0}^{m}=\frac{1}{2}$ is unstable, the shock layer will move away from $x_{0}^{m}=\frac{1}{2}$ to somewhere else, but not to the endpoints $x=0,1$. This suggests the existence of other stable equilibria for (3.33). For this example, it is easy to show that when $x_{0}^{m}=\frac{1}{2}$ is unstable (i.e., $\lambda_{0}\left(\frac{1}{2}\right)>0$ ), then $M\left(x_{0}\right)$ has exactly two more zeros that are symmetric about $x_{0}^{m}=\frac{1}{2}$. They correspond to two stable equilibrium values for $x_{0}(t)$. When $x_{0}^{m}=\frac{1}{2}$ is stable, then it is the only zero of $M\left(x_{0}\right)$. This analysis is illustrated by plotting $M\left(x_{0}\right)$ in Figure 5 .

TABLE 4a. A comparison of the asymptotic and numerical shock layer trajectories for (1.5) with $f(u)=u^{2} / 2$ and $g^{\prime}(x)=-1.9$. Here $\varepsilon=0.03, \mu=-1, d=0.5, \alpha=1$, and $x_{0}^{0}=0.205712482$.

\begin{tabular}{|c|c|c|c|}
\hline$t$ & $x_{0}(t)$ (asymptotic) & $x_{0}(t)$ (numerical) & error \\
\hline $.7466667 \times 10^{1}$ & .212682157 & .212676330 & $.582 \times 10^{-5}$ \\
$.5013333 \times 10^{2}$ & .236113084 & .236101298 & $.117 \times 10^{-4}$ \\
$.3754166 \times 10^{3}$ & .285065717 & .285059434 & $.628 \times 10^{-5}$ \\
$.3879890 \times 10^{4}$ & .352169342 & .352167776 & $.156 \times 10^{-5}$ \\
$.2523277 \times 10^{5}$ & .404404274 & .404403590 & $.683 \times 10^{-6}$ \\
$.4144176 \times 10^{6}$ & .465271835 & .465271222 & $.613 \times 10^{-6}$ \\
$.3061509 \times 10^{7}$ & .489469516 & .489468465 & $.105 \times 10^{-5}$ \\
$.8745862 \times 10^{7}$ & .496984897 & .496983905 & $.991 \times 10^{-6}$ \\
$.8407137 \times 10^{8}$ & .499999999 & .499999999 & $-.204 \times 10^{-9}$ \\
\hline
\end{tabular}

TABLE 4b. A comparison of the asymptotic and numerical shock layer trajectories for (1.5) with $f(u)=u^{2} / 2$ and $g^{\prime}(x)=-2.1$. Here $\varepsilon=0.03, \mu=-1, d=0.5, \alpha=1$, and $x_{0}^{0}=0.494999996$.

\begin{tabular}{|c|c|c|c|}
\hline$t$ & $x_{0}(t)$ (asymptotic) & $x_{0}(t)$ (numerical) & error \\
\hline $.2988174 \times 10^{4}$ & .494997385 & .494997382 & $.346 \times 10^{-8}$ \\
$.1725713 \times 10^{6}$ & .494847418 & .494847363 & $.548 \times 10^{-7}$ \\
$.4950768 \times 10^{7}$ & .489521616 & .489522250 & $-.633 \times 10^{-6}$ \\
$.7862987 \times 10^{7}$ & .486497884 & .486497678 & $.206 \times 10^{-6}$ \\
$.9804466 \times 10^{7}$ & .485206361 & .485204630 & $.173 \times 10^{-5}$ \\
$.1465816 \times 10^{8}$ & .483947388 & .483944120 & $.326 \times 10^{-5}$ \\
$.1659964 \times 10^{8}$ & .483811685 & .483908521 & $.316 \times 10^{-5}$ \\
$.2242408 \times 10^{8}$ & .483701958 & .483699155 & $.280 \times 10^{-5}$ \\
$.8697827 \times 10^{8}$ & .483689263 & .483686417 & $.284 \times 10^{-5}$ \\
\hline
\end{tabular}




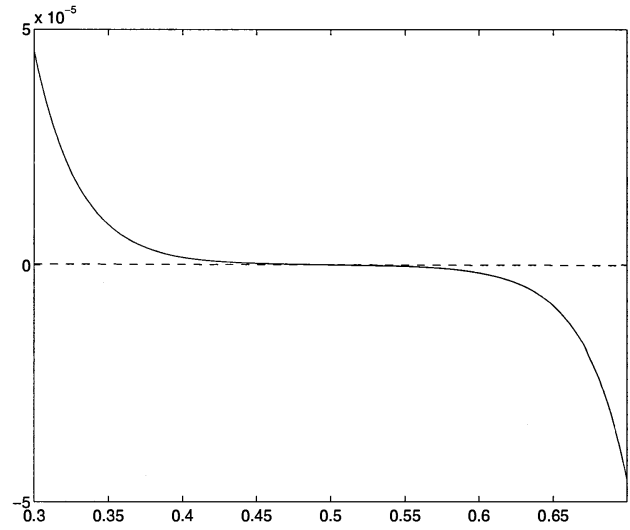

(a)

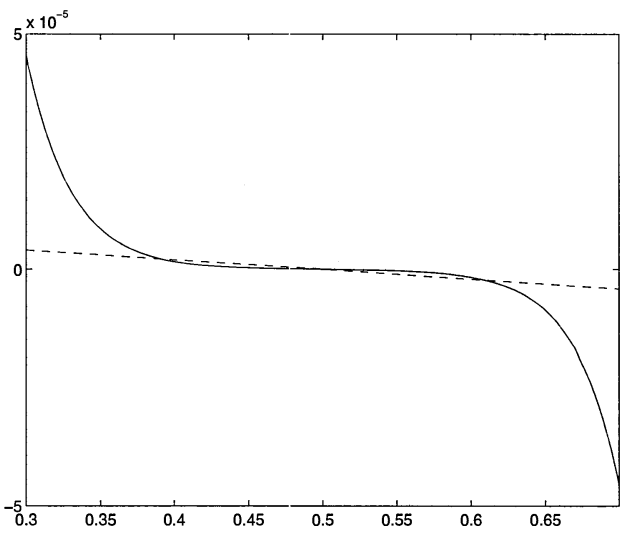

(b)

FIG. 3.1. Plots of $M_{1}\left(x_{0}\right) \equiv \alpha\left(-e^{-\alpha\left(1-x_{0}\right) / \varepsilon}+e^{-\alpha x_{0} / \varepsilon}\right)$ (solid lines) and $M_{2}\left(x_{0}\right) \equiv$ $\varepsilon^{\mu} e^{-d / \varepsilon} C\left(x_{0}-\frac{1}{2}\right)$ (dash lines) versus $x_{0}$ where $\alpha=1, \varepsilon=0.03, \mu=-1$, and $C=-2$. The intersection(s) of $M_{1}\left(x_{0}\right)$ and $M_{2}\left(x_{0}\right)$ is the zero(s) of $M\left(x_{0}\right)$. (a) $d=0.55$ : we have $\lambda_{0}\left(\frac{1}{2}\right)<0$, and the only equilibrium $x_{0}^{m}=\frac{1}{2}$ is stable; (b) $d=0.45$ : we have three zeros of $M\left(x_{0}\right)$ given by $x_{01}^{m} \approx 0.3902, x_{02}^{m}=0.5$, and $x_{03}^{m} \approx 0.6097$. Here $x_{02}^{m}=0.5$ is unstable and the rest are stable.

TABLE 5a. A comparison of the asymptotic and numerical shock layer trajectories for (1.5) with $f(u)=u^{2} / 2$ and $g^{\prime}(x)=-2$. Here $\varepsilon=0.03, \mu=-1, d=0.55, \alpha=1$, and $x_{0}^{0}=0.205646164$.

\begin{tabular}{|c|c|c|c|}
\hline$t$ & $x_{0}(t)$ (asymptotic) & $x_{0}(t)$ (numerical) & error \\
\hline $.1270000 \times 10^{2}$ & .216714648 & .216706869 & $.777 \times 10^{-5}$ \\
$.4765000 \times 10^{2}$ & .235153498 & .235142419 & $.110 \times 10^{-4}$ \\
$.3321062 \times 10^{3}$ & .281796238 & .281790749 & $.548 \times 10^{-5}$ \\
$.1271548 \times 10^{4}$ & .320208345 & .320206583 & $.176 \times 10^{-5}$ \\
$.1494813 \times 10^{5}$ & .392954938 & .392955455 & $-.517 \times 10^{-6}$ \\
$.1429582 \times 10^{6}$ & .457781790 & .457782599 & $-.808 \times 10^{-6}$ \\
$.5020859 \times 10^{6}$ & .488305699 & .488306411 & $-.712 \times 10^{-6}$ \\
$.1608928 \times 10^{7}$ & .499637394 & .499637666 & $-.271 \times 10^{-6}$ \\
$.5455925 \times 10^{8}$ & .500000000 & .499999999 & $.582 \times 10^{-9}$ \\
\hline
\end{tabular}

Therefore, there exists either one or two stable steady-state solutions of the form $u \sim u_{s}\left[\left(x-x_{0}^{m}\right) / \varepsilon\right]$ along the convergent nozzle we are considering. The analysis of [7] for the inviscid problem

$$
u_{t}+u u_{x}-c(x) u=0
$$

proved that standing shock waves in a convergent nozzle (i.e., $c(x)>0$ for all $x$ ) or in the convergent portion of a convergent-divergent nozzle are unstable. Our example has shown convincingly that the effect of viscosity and the boundary conditions in (1.5) allows for the existence of a stable standing wave in a convergent nozzle when $c(x)$ is replaced by the form in (1.7).

EXAMPLE 3.3. Next, we let $\alpha=1$ and consider a convergent-divergent nozzle where $g(x)=(x-a)^{2}$, and the constant $a$ satisfies $\frac{1}{2}<a<1$. Now the algebraic equation $2 g\left(x_{0}\right)-g(0)-g(1)=0$ has one root $x^{*} \in(0, a)$. If we choose $d$ so that 
TABLE 5b. A comparison of the asymptotic and numerical shock layer trajectories for (1.5) with $f(u)=u^{2} / 2$ and $g^{\prime}(x)=-2$. Here $\varepsilon=0.03, \mu=-1, d=0.45, \alpha=1$, and $x_{0}^{0}=0.494999513$.

\begin{tabular}{|c|c|c|c|}
\hline$t$ & $x_{0}(t)$ (asymptotic) & $x_{0}(t)$ (numerical) & error \\
\hline $.3895482 \times 10^{3}$ & .494967222 & .494967221 & $.946 \times 10^{-9}$ \\
$.7976059 \times 10^{4}$ & .494295191 & .494295125 & $.658 \times 10^{-7}$ \\
$.3291548 \times 10^{5}$ & .491389800 & .491389298 & $.502 \times 10^{-6}$ \\
$.8279433 \times 10^{5}$ & .480483494 & .480480756 & $.273 \times 10^{-5}$ \\
$.1491332 \times 10^{6}$ & .445338979 & .445330373 & $.860 \times 10^{-5}$ \\
$.1690847 \times 10^{6}$ & .429122276 & .429116007 & $.626 \times 10^{-5}$ \\
$.2249490 \times 10^{6}$ & .395546688 & .395541088 & $.560 \times 10^{-5}$ \\
$.3072491 \times 10^{6}$ & .390353158 & .390348796 & $.436 \times 10^{-5}$ \\
$.2905679 \times 10^{10}$ & .390284916 & .390281195 & $.372 \times 10^{-5}$ \\
\hline
\end{tabular}

$0<d<x^{*}$, then it is easy to see that for $\varepsilon \rightarrow 0$ the function $M\left(x_{0}\right)$ in (3.33) will have three zeros $x_{01}^{m}, x_{02}^{m}$, and $x_{03}^{m}$, satisfying $0<x_{01}^{m}<x_{02}^{m}<x^{*}$ and $a<x_{03}^{m}<1$. These zeros are illustrated in Figure 6 for the parameter values $\varepsilon=0.04, \alpha=1, \mu=-1$, $d=0.171244968$, and $a=0.8$. From the discussion following Corollary 3.4, it is clear that $x_{01}^{m}$ and $x_{03}^{m}$ are stable equilibria and that $x_{02}^{m}$ is unstable. In Figure 7, we verify the stability of $x_{01}^{m}$ and $x_{03}^{m}$ by plotting the numerical solution to (1.5) at different times for two initial values $x_{0}^{0}$. The two initial values $x_{0}^{0}$ in Figures $7 \mathrm{a}$ and $7 \mathrm{~b}$ are so close that there is only one unstable equilibrium for $x_{0}(t)$ between them, which is $x_{02}^{m}$.

EXAmple 3.4. Finally, we give an example to illustrate that it is possible to construct a nozzle geometry to guarantee an arbitrary number of steady state internal layer solutions. We take $g(x)=\sin (n \pi x)$ where $n$ is positive integer. Let $x_{i}^{*}=i / n$ for $i=0, \ldots, n$ be the $i$-th zero of $g(x)$. In this case, the differential equation (3.26) for $x_{0}(t)$ becomes

$$
x_{0}^{\prime} \sim \alpha\left[e^{-\alpha x_{0} / \varepsilon}-e^{-\alpha\left(1-x_{0}\right) / \varepsilon}\right]-\varepsilon^{\mu} e^{-d / \varepsilon} \zeta \sin \left(n \pi x_{0}\right), \quad \zeta=1-\frac{n^{2} \pi^{4} \varepsilon^{2}}{6 \alpha^{2}}+\cdots
$$

It is easy to see that if $d$ is chosen such that $0<d<x_{1}^{*}$, then for $\varepsilon \rightarrow 0$, there exists $N \equiv n+(n-1) \bmod 2$ equilibria $x_{0 i}^{m}, i=1,2, \ldots, N$. Among these equilibria, $x_{0 i}^{m}$ for $i=1,3, \ldots, N$ are stable and the rest are unstable. Note that if $d \geq x_{1}^{*}$, then the number of the equilibria may be less than $N$.

We now implement a numerical experiment to illustrate this analysis. We choose $n=4, \alpha=1, \mu=0, d=0.19$, and $\varepsilon=0.02$. In this case, we have three stable equilibria at $x_{01}^{m} \approx 0.2044, x_{03}^{m}=0.5$, and $x_{05}^{m} \approx 0.7955$, and two unstable equilibria at $x_{02}^{m} \approx 0.2440$ and $x_{04}^{m} \approx 0.7559$, which we compute from (3.36) using a two-term expansion for $\zeta$. We choose the initial values $x_{0}^{0}=0.24$ and $x_{0}^{0}=0.25$ when computing the numerical solution to (1.5). The asymptotic and numerical results are shown in Tables $6 \mathrm{a}$ and $6 \mathrm{~b}$. These tables also display the asymptotic result (3.36) with both the one-term and the two-term expansions for $\zeta$. The error terms in the fourth and sixth columns represent the difference between the asymptotic and numerical results. Since the higher order terms in $\zeta$ in (3.36) are significant, we observe from Table 6 that a two-term expansion for $\eta$ is certainly needed to obtain close quantitative agreement with the numerical results for $x_{0}(t)$. Finally, in Figure 8, we plot the shock layer 


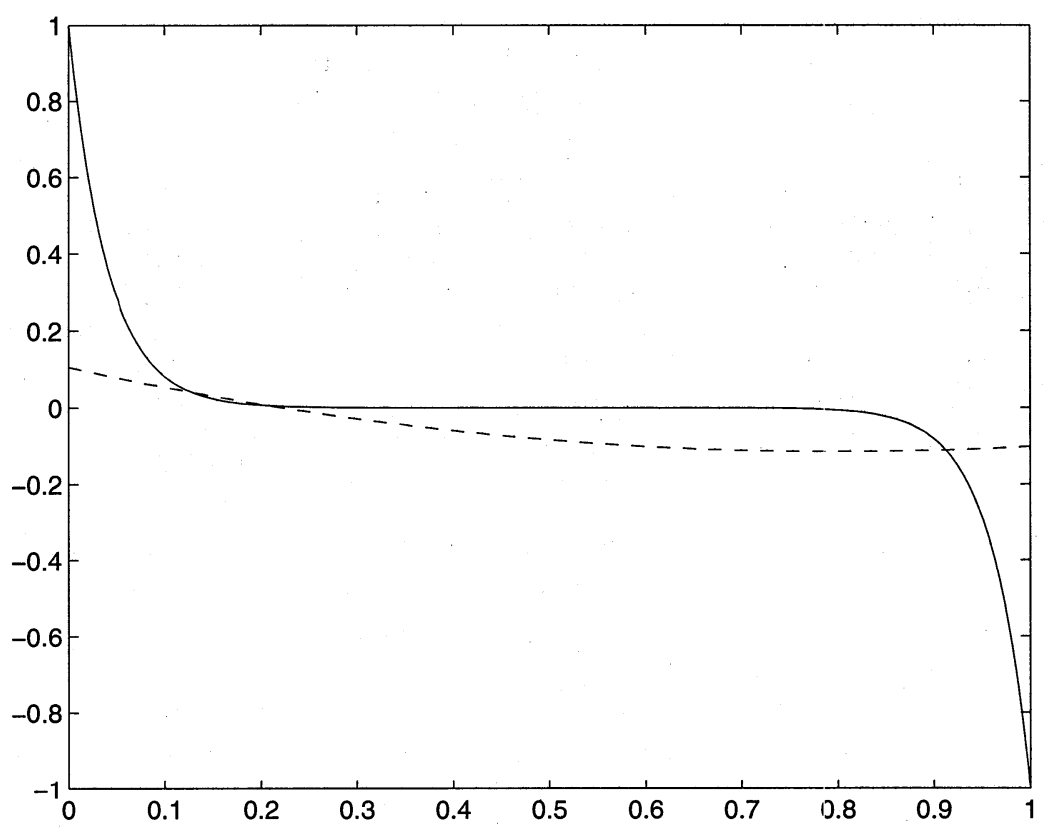

Fig. 3.2. Plots of $M_{1}\left(x_{0}\right) \equiv \alpha\left(-e^{-\alpha\left(1-x_{0}\right) / \varepsilon}+e^{-\alpha x_{0}} / \varepsilon\right)$ (solid lines) and $M_{2}\left(x_{0}\right) \equiv$ $\varepsilon^{\mu} e^{-d / \varepsilon}\left(\left(x_{0}-a\right)^{2}-\frac{1}{2}\left[a^{2}+(1-a)^{2}\right]+\pi^{2} \alpha^{-2} \varepsilon^{2} / 3\right)$ versus $x_{0}$ where $\varepsilon=0.04, a=0.8, \alpha=1$, $\mu=-1$, and $d=0.1712 \ldots$. The intersections of $M_{1}\left(x_{0}\right)$ and $M_{2}\left(x_{0}\right)$, which are the zeros of $M\left(x_{0}\right)$, are $x_{01}^{m} \approx 0.1286, x_{02}^{m} \approx 0.2077$ and $x_{03}^{m} \approx 0.9122$. Here the equilibrium $x_{02}^{m}$ is unstable, and the other two are stable.

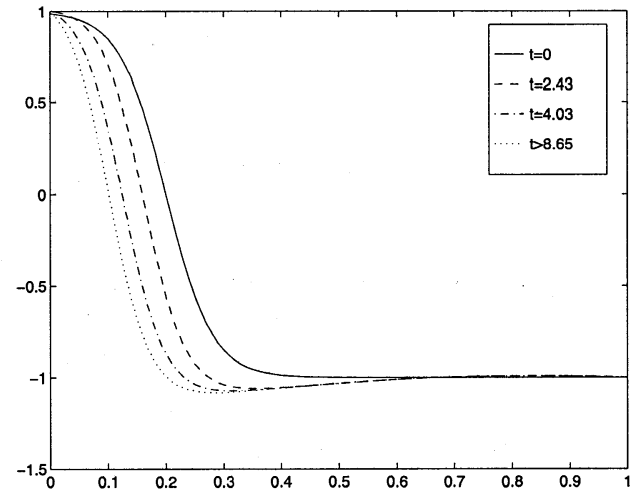

(a)

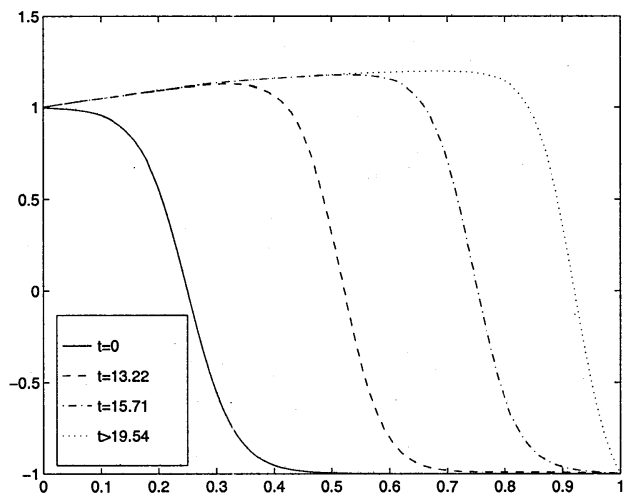

(b)

FIG. 3.3. Plots of the numerical solutions to (1.5) at different times with $f(u)=u^{2} / 2$ and $g(x)=(x-a)^{2}$ where $\varepsilon=0.04, a=0.8, \alpha=1, \mu=-1, d=0.1712 \ldots$ and initial condition $u_{0}(x)=u_{s}\left[\left(x-x_{0}^{0}\right) / \varepsilon\right]$. (a) $x_{0}^{0}=0.2$ : shock layer moves towards left; (b) $x_{0}^{0}=0.25$ : shock layer moves towards right.

evolution corresponding to the data in Table 6, which shows that $x_{01}^{m}$ and $x_{03}^{m}$ are stable equilibria, while $x_{02}^{m}$ is an unstable equilibrium.

Appendix A. Derivation of equation (1.1). Consider the Ginzburg-Landau equation (1.1) with Neumann boundary condition in a cylinder of revolution with 
TABLE 6a. A comparison of the asymptotic and numerical shock layer trajectories for (1.5) with $f(u)=u^{2} / 2$ and $g(x)=\sin (4 \pi x)$. Here $\varepsilon=0.02, \alpha=1, \mu=0$, $d=0.19$, and $x_{0}^{0}=0.239991525$.

\begin{tabular}{|c|c|c|c|c|c|}
\hline$t$ & $x_{0}(t)$ (num.) & $(3.36)$ 1-term & error1 & $(3.36) 2$-term & error2 \\
\hline $.1133545 \times 10^{3}$ & .239718703 & .239610666 & $-.108 \times 10^{-3}$ & .239726721 & $.801 \times 10^{-5}$ \\
$.5512484 \times 10^{3}$ & .238497828 & .237864222 & $-.633 \times 10^{-3}$ & .238543918 & $.460 \times 10^{-4}$ \\
$.1135106 \times 10^{4}$ & .236387221 & .234724657 & $-.166 \times 10^{-2}$ & .236505117 & $.117 \times 10^{-3}$ \\
$.2580156 \times 10^{4}$ & .228182044 & .222194243 & $-.598 \times 10^{-2}$ & .228599741 & $.417 \times 10^{-3}$ \\
$.4255100 \times 10^{4}$ & .214978265 & .206459679 & $-.851 \times 10^{-2}$ & .215700702 & $.722 \times 10^{-3}$ \\
$.6061413 \times 10^{4}$ & .206403299 & .201517420 & $-.488 \times 10^{-2}$ & .206902782 & $.499 \times 10^{-3}$ \\
$.8360356 \times 10^{4}$ & .204368747 & .200989316 & $-.337 \times 10^{-2}$ & .204672929 & $.304 \times 10^{-3}$ \\
$.1606592 \times 10^{5}$ & .204187662 & .200966538 & $-.322 \times 10^{-2}$ & .204461290 & $.273 \times 10^{-3}$ \\
$.6239090 \times 10^{5}$ & .204187642 & .200966538 & $-.322 \times 10^{-2}$ & .204461248 & $.273 \times 10^{-3}$ \\
\hline
\end{tabular}

TABLE 6b. A comparison of the asymptotic and numerical shock layer trajectories for (1.5) with $f(u)=u^{2} / 2$ and $g(x)=\sin (4 \pi x)$. Here $\varepsilon=0.02, \alpha=1, \mu=0$, $d=0.19$, and $x_{0}^{0}=0.250022546$.

\begin{tabular}{|c|c|c|c|c|c|}
\hline$t$ & $x_{0}(t)$ (num.) & $(3.36) 1$-term & error1 & $(3.36) 2$-term & error2 \\
\hline $.9389256 \times 10^{2}$ & .250384983 & .250386816 & $.183 \times 10^{-5}$ & .250384917 & $-.664 \times 10^{-7}$ \\
$.5804413 \times 10^{3}$ & .252677437 & .252755503 & $.780 \times 10^{-4}$ & .252672223 & $-.521 \times 10^{-5}$ \\
$.1650848 \times 10^{4}$ & .261513735 & .262616867 & $.110 \times 10^{-2}$ & .261438939 & $-.748 \times 10^{-4}$ \\
$.3207804 \times 10^{4}$ & .296383726 & .306534177 & $.101 \times 10^{-1}$ & .295721003 & $-.662 \times 10^{-3}$ \\
$.4969111 \times 10^{4}$ & .398142523 & .424482167 & $.263 \times 10^{-1}$ & .396102752 & $-.204 \times 10^{-2}$ \\
$.6545529 \times 10^{4}$ & .469337132 & .481526941 & $.121 \times 10^{-1}$ & .468203669 & $-.113 \times 10^{-2}$ \\
$.9610786 \times 10^{4}$ & .497704786 & .498961715 & $.125 \times 10^{-2}$ & .497567194 & $-.137 \times 10^{-3}$ \\
$.1570298 \times 10^{5}$ & .499987099 & .499996630 & $.953 \times 10^{-5}$ & .499985677 & $-.142 \times 10^{-5}$ \\
$.6945472 \times 10^{6}$ & .5 & .5 & $.573 \times 10^{-9}$ & .5 & $-.274 \times 10^{-9}$ \\
\hline
\end{tabular}

cross-section described in dimensional variables by $R=R_{0} F(X / L)$ (see Figure 1.1):

(A.1a)

$$
\begin{aligned}
& U_{t}=D\left(U_{R R}+R^{-1} U_{R}+U_{X X}\right)+Q_{0} Q(U), \quad 0<X<L, \quad 0<R<R_{0} F(X / L), \\
& \text { (A.1b) } \quad\left(U_{R}, U_{X}\right) \cdot\left(1,-L^{-1} R_{0} F^{\prime}(X / L)=0, \quad \text { on } R=R_{0} F(X / L)\right. \text {, } \\
& U_{X}=0, \quad \text { on } X=0, L \text {. }
\end{aligned}
$$

Here $D$ and $Q_{0}$ are positive constants and $Q(U)$ is described following (1.1). We assume that the cylinder is long and thin so that $R_{0} \ll L$. In terms of the dimensionless variables $r=R_{0}^{-1} R, x=L^{-1} X$, and $\tau=D L^{-2} t$, (A.1) becomes

$$
\begin{gathered}
U_{\tau}=\delta^{-2}\left(U_{r r}+r^{-1} U_{r}\right)+U_{x x}+\bar{Q} Q(U), \quad 0<x<1, \quad 0<r<F(x), \\
U_{r}-\delta^{2} U_{x} F^{\prime}(x)=0, \quad \text { on } \quad r=F(x) \\
U_{x}=0, \quad \text { on } \quad x=0,1 .
\end{gathered}
$$

Here $\delta=R_{0} / L \ll 1$ and $\bar{Q}=D^{-1} Q_{0} L^{2}$.

We now derive a partial differential equation that is valid for $U$ in the limit $\delta \rightarrow 0$. We expand $U$ away from the endpoints at $x=0,1$, as $U=U_{0}+\delta^{2} U_{1}+\cdots$. Substituting this expansion into (0.2a) and (0.2b) and collecting powers of $\delta^{2}$, we obtain

$$
U_{0 r r}+r^{-1} U_{r}=0, \quad \text { in } 0<r<F(x) ; \quad U_{0 r}=0, \quad \text { on } r=F(x),
$$




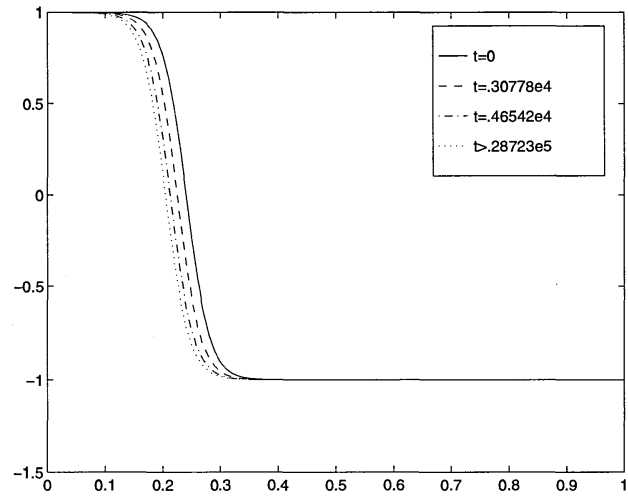

(a)

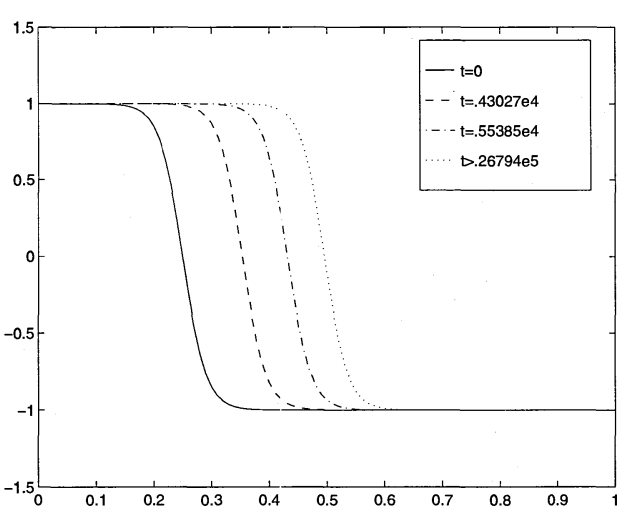

(b)

FIG. 3.4. Plots of the numerical solutions to (1.5) at different times with $f(u)=u^{2} / 2$ and $g(x)=\sin (4 \pi x)$ where $\varepsilon=0.02, \alpha=1, \nu=0, d=0.19$, and initial condition $u_{0}(x)=u_{s}\left[\left(x-x_{0}^{0}\right) / \varepsilon\right]$. (a) $x_{0}^{0}=0.24$ : shock layer moves towards left; (b) $x_{0}^{0}=0.25$ : shock layer moves towards right.

and

$$
\begin{aligned}
U_{1 r r}+r^{-1} U_{1 r} & =U_{0 \tau}-\bar{Q} Q\left(U_{0}\right)-U_{0 x x}, \quad \text { in } 0<r<F(x), \\
U_{1 r} & =F^{\prime}(x) U_{0 x}, \quad \text { on } r=F(x) .
\end{aligned}
$$

The first equation gives $U_{0}=U_{0}(x, t)$. To determine an evolution equation for $U_{0}$, we write $(0.4 \mathrm{a})$ as $\left(r U_{1 r}\right)_{r}=r\left(U_{0 \tau}-\bar{Q} Q\left(U_{0}\right)-U_{0 x x}\right)$. Integrating this equation with respect to $r$ from 0 to $F(x)$ and applying the boundary condition $(0.4 \mathrm{~b})$, we get

$$
U_{0 \tau}=U_{0 x x}+2 F^{-1} F^{\prime} U_{0 x}+\bar{Q} Q\left(U_{0}\right) .
$$

Let $A$ denote the cross-sectional area of the domain, so that $A=\pi F^{2}$. Then, from $(0.5)$ and $(0.2 \mathrm{c})$, we get the one-dimensional reaction-diffusion equation

$$
\begin{gathered}
U_{0 \tau}=\frac{1}{A}\left(A U_{0 x}\right)_{x}+\bar{Q} Q\left(U_{0}\right), \quad 0<x<1, \quad t>0, \\
U_{0 x}(0, \tau)=U_{0 x}(1, \tau)=0 .
\end{gathered}
$$

To study the slow motion of internal layers under (0.6), we suppose $\bar{Q} \gg 1$ and so we write $\bar{Q}=\varepsilon^{-2}$ for some $\varepsilon \ll 1$. Then, setting $t=\varepsilon^{-2} \tau$, we find that (0.6) reduces to (1.1) when the cross-sectional area $A=A(x, \varepsilon)$ is given by $A(x, \varepsilon)=1+\varepsilon^{\mu} g(x) e^{-d / \varepsilon}$.

\section{REFERENCES}

[1] U. Ascher, R. Christiansen, And R. Russell, Collocation software for boundary value ODE's, Math. Comp., 33 (1979), pp. 659-679.

[2] U. Ascher, R. Mattheis, And R. Russell, Numerical Solution of Boundary Value Problems for Ordinary Differential Equations, Prentice Hall, Englewood Cliffs, NJ, 1988.

[3] J. R. CASH, The integration of stiff initial value problems in ODEs using modified extended backward differentiation formulae, Comput. Math. Appl., 9 (1983), pp. 645-657.

[4] J. CARR AND R. PEgo, Metastable patterns in solutions of $u_{t}=\varepsilon^{2} u_{x x}-f(u)$, Comm. Pure Appl. Math., 42 (1989), pp. 523-576.

[5] R. G. CASTEN AND C. J. HOLLAND, Instability results for reaction diffusion equations with Neumann boundary conditions, J. Diff. Equat., 27 (1978), pp. 266-273.

[6] S. J. Chapman AND G. RichaRdson, Vortex pinning by inhomogeneities in type-2 superconductors, Physica D, 108 (1997), pp. 397-407. 
[7] P. Embid, J. Goodman, AND A. MAJDA, Multiple steady states for 1-D transonic flow, SIAM J. Sci. Statist. Comput., 5 (1984), pp. 21-41.

[8] G. FuSCO AND J. K. HALE, Slow motion manifolds, dormant instability and singular perturbations, J. Dyn. Diff. Equat., 1 (1989), pp. 75-94.

[9] P. Grindrod, M. A. Lewis, And J. D. Murray, A geometrical approach to wave-type solutions of excitable reaction-diffusion systems, Proc. Roy. Soc. London, Series A, Vol. 433, No. 1887 (1991), pp. 151-164.

[10] F. A. Howes, Some stability results for advection-diffusion equations, Stud. Appl. Math., 74 (1986), pp. 35-53.

[11] S. HSU AND T. P. LIU, Nonlinear singular sturm-liouville problems and an application to transonic flow through a nozzle, Comm. Pure Appl. Math., 43 (1990), pp. 31-61.

[12] S. JIMBo AND Y. MoRITA, Stable solutions with zeros to the Ginzburg-Landau equation with Neumann boundary condition, J. Diff. Equat., 128 (1996), pp. 596-613.

[13] G. KREISS AND H. KREISS, Convergence to steady state of solutions of Burgers equation, Appl. Numerical Math., 2 (1986), pp. 161-179.

[14] J. LAFORGUE, Using exponential asymptotics to study the metastability and supersensitivity of shock and transition layers, University of Washington, Ph.D. Thesis, 1994.

[15] J. LAFORGUE AND R. E. O'MALLEY, On the motion of viscous shocks and the super-sensitivity of their steady-state limits, Methods and Appl. of Anal., 1 (1994), pp. 465-487.

[16] F. H. LIN AND Q. DU, Ginzburg-landau vortices: dynamics, pinning and hysteresis, SIAM J. Math. Anal., 28 (1997), pp. 1265-1293.

[17] T. P. LIU, Nonlinear stability and instability of transonic flows through a nozzle, Comm. Math. Phys., 83 (1982), pp. 243-260.

[18] H. MATANO, Asymptotic behavior and stability of solutions of semilinear diffusion equations, Publ. RIMS, Kyoto Univ., 15 (1979), pp. 401-454.

[19] L. G. REYNA AND M. J. WARD, On the exponentially slow motion of a viscous shock, Comm. Pure Appl. Math., 48 (1995), pp. 79-120.

[20] J. S. Scroggs AND D. C. SoRENSEN, An asymptotic induced numerical method for the convection-diffusion-reaction equation, in Lecture Notes in Pure and Appl. Math. 120, 1989, pp. 81-114.

[21] X. SUn AND M. J. WARD, Metastability for a generalized Burgers equation with applications to propagating flame-fronts, European J. Appl. Math., 10 (1999), pp. 27-53.

[22] M. J. WARD, Metastable patterns, layer collapses, and coarsening for a one-dimensional Ginzburg-Landau equation, Stud. Appl. Math., 91 (1994), pp. 51-93.

[23] — Dynamic metastability and singular perturbations, in Boundaries, Interfaces, and Transitions, Michel C. Delfour, ed., Banff 95, CRM Proc. Lecture Notes 13, AMS, Providence, R.I., 1998, pp. 237-263. 
